\title{
NUMERICAL METHODS FOR TWO-PARAMETER LOCAL BIFURCATION ANALYSIS OF MAPS*
}

\author{
W. GOVAERTS ${ }^{\dagger}$, R. KHOSHSIAR GHAZIANI ${ }^{\ddagger}$, YU. A. KUZNETSOV $^{\S}$, AND \\ H. G. E. MEIJER
}

\begin{abstract}
We discuss new and improved algorithms for the bifurcation analysis of fixed points and periodic orbits (cycles) of maps and their implementation in MATCONT, a MATLAB toolbox for continuation and bifurcation analysis of dynamical systems. This includes the numerical continuation of fixed points of iterates of the map with one control parameter, detecting and locating their bifurcation points (i.e., limit point, period-doubling, and Neimark-Sacker) and their continuation in two control parameters, as well as detection and location of all codimension 2 bifurcation points on the corresponding curves. For all bifurcations of codim 1 and 2, the critical normal form coefficients are computed, both numerically with finite directional differences and using symbolic derivatives of the original map. Using a parameter-dependent center manifold reduction, explicit asymptotics are derived for bifurcation curves of double and quadruple period cycles rooted at codim 2 points of cycles with arbitrary period. These asymptotics are implemented into the software and allow one to switch at codim 2 points to the continuation of the double and quadruple period bifurcations. We provide two examples illustrating the developed techniques: a generalized Hénon map and a juvenile/adult competition model from mathematical biology.
\end{abstract}

Key words. bifurcations of fixed points, cycles, normal forms, branch switching

AMS subject classifications. 34C20, 37G05, 65P30

DOI. $10.1137 / 060653858$

1. Introduction. Discrete-time dynamical systems generated by iterated maps appear in many scientific areas, such as economics, engineering, and ecology. The usual method for getting an insight into their behavior is to compute many orbits, starting from various initial points (i.e., simulation). Another tool for gaining a better understanding of these systems is bifurcation analysis. It provides theoretic results on the classification of possible modes of behavior, which may explain results of the simulations at different values of control parameters. The application of such theoretical results requires robust numerical computational tools, as a purely symbolic approach is often futile. In the present paper we describe how CL_MATCONT, which supports only ODEs $[7,8]$, has been complemented by the addition of the new package CL_MATCONTM, which supports local bifurcation analysis of generic finite-dimensional smooth maps. The software and a manual are freely available at http://www.matcont.ugent.be/. Before discussing and comparing existing computer tools for maps, we fix some notation and discuss some events which any standard

*Received by the editors March 7, 2006; accepted for publication (in revised form) April 4, 2007; published electronically November 2, 2007.

http://www.siam.org/journals/sisc/29-6/65385.html

${ }^{\dagger}$ Department of Applied Mathematics and Computer Science, Ghent University, Krijgslaan 281S9, B-9000 Gent, Belgium (Willy.Govaerts@UGent.be).

${ }^{\ddagger}$ Department of Applied Mathematics and Computer Science, Ghent University, Krijgslaan 281S9, B-9000 Gent, Belgium, and Department of Mathematics, Faculty of Science, Shahrekord University, P.O. Box 115, Shahrekord, Iran (Reza.KhoshsiarGhaziani@UGent.be).

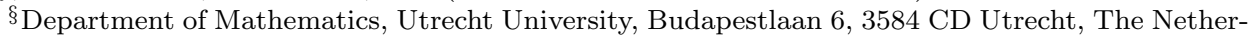
lands, and Institute of Mathematical Problems of Biology, Russian Academy of Sciences, Pushchino, Moscow Region, 142290 Russia (kuznet@math.uu.nl).

IDepartment of Electrical Engineering, Mathematics and Computer Science, Universiteit Twente, P.O. Box 217, 7500 AE Enschede, The Netherlands (meijerhge@math.utwente.nl). 
software should be able to handle.

Consider a map

$$
x \mapsto f(x, \alpha),
$$

where $x \in \mathbb{R}^{n}$ is a state variable vector and $\alpha \in \mathbb{R}^{p}$ is a parameter vector. We assume that $f$ is sufficiently smooth so that all partial derivatives below are well defined. Write the $K$ th iterate of (1.1) at some parameter value as

$$
x \mapsto f^{(K)}(x, \alpha), \quad f^{(K)}: \mathbb{R}^{n} \times \mathbb{R}^{p} \rightarrow \mathbb{R}^{n},
$$

where

$$
g(x, \alpha):=f^{(K)}(x, \alpha)=\underbrace{f(f(f(\cdots f}_{K \text { times }}(x, \alpha), \alpha), \alpha), \alpha) .
$$

The bifurcation analysis of (1.1) usually starts with fixed points. Numerically we continue fixed points of this map, i.e., solutions to the equation

$$
F(x, \alpha) \equiv f(x, \alpha)-x=0,
$$

with one control parameter.

While varying one parameter, one may encounter codim 1 bifurcations of fixed points, i.e., critical parameter values where the stability of the fixed point changes. The eigenvalues of the Jacobian matrix of $f$ are called multipliers. The fixed point is asymptotically stable if $|\lambda|<1$ for every multiplier $\lambda$. If there exists a multiplier $\lambda$ with $|\lambda|>1$, then the fixed point is unstable. While following a curve of fixed points, three codim 1 singularities related to stability changes can generically occur, namely a limit point (fold, LP), a period-doubling (flip, PD), and a Neimark-Sacker (NS) point; see section 2 for details. Encountering such a bifurcation, one may use the formulas for the normal form coefficients derived via the center manifold reduction (see, e.g., [18, section 5.4]) to analyze the bifurcation. A nongeneric situation occurs at a branch point (BP) where the Jacobian matrix $\left[F_{x}(x, \alpha), F_{\alpha}(x, \alpha)\right]$ of $(1.4)$ is rank deficient. Here the implicit function theorem cannot be applied to ensure the existence of a unique smooth branch of solutions. However, it is encountered often in practical problems that exhibit some form of symmetry (equivariance).

When two control parameters are allowed to vary, eleven codim 2 bifurcations can be met in generic families of maps (1.1), where curves of codim 1 bifurcations intersect or meet tangentially. The critical normal form coefficients for all generic codim 2 bifurcation points have been derived earlier in [19, 20] using a combined reduction-normalization technique. Note that the full verification of the genericity of a codim 2 bifurcation requires not only establishing its nondegeneracy at the critical parameter values but also a careful analysis of how the system depends on parameters. This "transversality" issue, which includes the expression of the canonical unfolding parameters of the normal form in terms of the original map parameters, has received little attention in the literature to date. When available, this information could be used to approximate codim 1 bifurcation curves that emanate from the codim 2 points.

There are several standard software packages supporting bifurcation analysis of iterated maps. Orbits of maps and one-dimensional invariant manifolds of saddle fixed points can be computed and visualized using DYNAMics [24] and DsTool [3]. Location and continuation of fixed-point bifurcations is implemented in AUTO [9] 
and the LBFP-version of LOCBIF [15]. The latter program computes the critical normal form coefficient at LP points and locates some codim 2 bifurcations along branches of codim 1 fixed points and cycles. CONTENT [17] was the first software that computed the critical normal form coefficients for all three codim 1 bifurcations of fixed points and cycles and allowed one to continue these bifurcations in two parameters and to detect all eleven codim 2 singularities along them. Branch switching at PD and BP points is also implemented in AUTO, LOCBIF, and CONTENT. However, only trivial branch switching is possible at codim 2 points and only for two (cusp and 1:1 resonance) of eleven codim 2 bifurcations are the critical normal form coefficients computed by the current version of CONTENT. No software supports switching at codim 2 points to the continuation of the double- and quadruple-period bifurcation curves. In the present paper we describe how CL_MATCONTM continues fixed points of an iterate of a map and handles the above-mentioned bifurcations.

The paper is organized as follows. In section 2, we first discuss continuation of fixed points of the iterated map (1.2) and detection of fold, flip, and NS bifurcations and branch points. After a short discussion of all generic codim 1 bifurcations of period- $K$ orbits, we present the continuation of fold, flip, and NS curves of a cycle and detection of codim 2 points along these curves. The algorithms used here are similar to those implemented in CONTENT [12]. In section 3, we provide a (parameterdependent) normal form for each codim 2 bifurcation point of period- $K$ orbits in the minimal possible phase dimension. Then we consider the branch switching at codim 2 points, where we use parameter-dependent center-manifold reduction. In section 4 we present some algorithmic and numerical details, including the computation of partial derivatives of (1.2) up to and including order 5, using both symbolic partial derivatives of (1.1) and finite differences. In section 5 we demonstrate the use of the algorithms and implementation. Finally, in section 6 we draw some conclusions and mention a few problems for future work.

2. Continuation and detection for codim 1 bifurcations of fixed points and cycles.

2.1. Defining the system and singularities for cycles. Assume that for some $\alpha=\alpha_{0}$ and $K \geq 1$ the map $g$ has a fixed point $x=x_{0}$, which is not a fixed point of $f^{(J)}\left(\cdot, \alpha_{0}\right)$ for $1 \leq J<K$ when $K>1$. In other words, $x_{0}$ is a fixed point or a cycle with minimal period $K$ of $f\left(\cdot, \alpha_{0}\right)$.

Each point $x$ of the cycle of period $K$ then satisfies the fixed point equation for the $K$ th iterate

$$
g(x, \alpha)-x=0
$$

As in CONTENT, branches of period- $K$ cycles are computed in CL_MATCONTM by a variant of the Gauss-Newton continuation algorithm [1] applied to $(2.1)$; see [7, 8].

If the Jacobian matrix $A=g_{x}\left(x_{0}, \alpha_{0}\right)$ has no eigenvalue $\lambda$ with $|\lambda|=1$, i.e., for a hyperbolic fixed point, the dynamics near the origin is topologically equivalent to that of the linear map $x \mapsto A x$ by the Grobman-Hartman theorem. If eigenvalues with $|\lambda|=1$ are present, the center manifold theorem guarantees the existence of local stable, unstable, and center invariant manifolds near the fixed point for parameter values close to $\alpha_{0}$. Exact formulations of these theorems, together with references to the original papers, can be found in any standard book on dynamical systems, e.g., [2]. On the stable and unstable manifolds, the local dynamics is still determined by the linear part of the map. In contrast, the dynamics in the center manifolds depends on 
both linear and nonlinear terms. Not all nonlinear terms are equally important, since some of them can be eliminated by an appropriate smooth coordinate transformation that depends smoothly on parameters and that puts the map restricted to the center manifold into a normal form, at least up to some order. Nonhyperbolic fixed points bifurcate; i.e., the dynamics near such points changes topologically under parameter variations. The birth of extra invariant objects, such as cycles or tori, is described by a parameter-dependent normal form of the restriction of $g$ to a center manifold. Even though neither the center manifold nor the normal form on it is unique, the qualitative conclusions do not depend on the choices that are made [2, p. 266].

For the computations of the center manifold we write the Taylor expansion of $g$ about $\left(x_{0}, \alpha_{0}\right)$ :

$$
\begin{aligned}
g\left(x_{0}+x, \alpha_{0}+\alpha\right)= & x_{0}+A x+\frac{1}{2} B(x, x)+\frac{1}{6} C(x, x, x) \\
& +\frac{1}{24} D(x, x, x, x)+\frac{1}{120} E(x, x, x, x, x) \\
& +J_{1} \alpha+\frac{1}{2} J_{2}(\alpha, \alpha) \\
& +A_{1}(x, \alpha)+\frac{1}{2} B_{1}(x, x, \alpha)+\frac{1}{6} C_{1}(x, x, x, \alpha)+\frac{1}{24} D_{1}(x, x, x, x, \alpha) \\
& +\frac{1}{2} A_{2}(x, \alpha, \alpha)+\frac{1}{4} B_{2}(x, x, \alpha, \alpha)+\frac{1}{12} C_{2}(x, x, x, \alpha, \alpha) \\
& +\cdots,
\end{aligned}
$$

where all functions are multilinear forms of their arguments and the dots denote higher order terms in $x$ and $\alpha$. In particular, $A=g_{x}\left(x_{0}, \alpha\right)$, and the components of the multilinear functions $B$ and $C$ are given by

$$
B_{i}(u, v)=\sum_{j, k=1}^{n} \frac{\partial^{2} g_{i}\left(x_{0}, \alpha_{0}\right)}{\partial x_{j} \partial x_{k}} u_{j} v_{k}, \quad C_{i}(u, v, w)=\sum_{j, k, l=1}^{n} \frac{\partial^{3} g_{i}\left(x_{0}, \alpha_{0}\right)}{\partial x_{j} \partial x_{k} \partial x_{l}} u_{j} v_{k} w_{l},
$$

for $i=1,2, \ldots, n$. From now on, $I_{n}$ is the unit $n \times n$ matrix and $\|x\|=\sqrt{\langle x, x\rangle}$, where $\langle u, v\rangle=\bar{u}^{T} v$ is the standard scalar product in $\mathbb{C}^{n}\left(\right.$ or $\left.\mathbb{R}^{n}\right)$.

Generically, the following events happen in the state space near a bifurcation (see, for example, [18]). When the control parameter crosses the critical value corresponding to a fold (LP) bifurcation, two fixed points of $g$ collide and disappear, provided that $a_{1} \neq 0$. This implies the collision of two period- $K$ cycles of the original map $f$. When the control parameter crosses the critical value corresponding to a flip (PD) bifurcation and $b_{1} \neq 0$, a cycle of period 2 for $g$ bifurcates from the fixed point, which is a cycle of period $2 K$ for map $f$. Finally, provided that $c_{1}=\Re\left(d_{1}\right) \neq 0$, a unique closed invariant curve for $g$ around the fixed point appears on the center manifold, when the control parameter crosses the critical value corresponding to the Neimark-Sacker (NS) bifurcation. For the original map, this means the appearance of $K$ disjoint curves, cyclically shifted by $f$.

To detect the aforementioned bifurcations, as well as branch points of (2.1), we use in CL_MATCONTM the standard test functions listed in Table 2.1, where $t$ is the tangent vector to the curve (2.1) in the $X$-space for $X=(x, \alpha)^{T}, F(X)=g(x, \alpha)-x$, $A=g_{x}$ is the Jacobian matrix of $g=f^{(K)}$, and $\odot$ is the bialternate matrix product; see, e.g., [13, section 4.4.4]. We notice that $\operatorname{det}\left(A \odot A-I_{m}\right)$, where $m=\frac{1}{2} n(n-1)$, 
TABLE 2.1

Detection of codim 1 bifurcations of cycles.

\begin{tabular}{|c|c|}
\hline \hline Bifurcation & Test function(s) \\
\hline \hline LP & $t_{n+1}=0,\left(\begin{array}{c}F_{X} \\
t^{T}\end{array}\right) \neq 0$ \\
\hline $\mathrm{PD}$ & $\operatorname{det}\left(A+I_{n}\right)=0$ \\
\hline $\mathrm{NS}$ & $\operatorname{det}\left(A \odot A-I_{m}\right)=0$ \\
\hline $\mathrm{BP}$ & $\operatorname{det}\left(\begin{array}{c}F_{X} \\
t^{T}\end{array}\right)=0$ \\
\hline \hline
\end{tabular}

also vanishes at neutral saddles. We distinguish true NS bifurcations from neutral saddles when processing the NS points.

2.2. Defining systems and singularities for codim 1 bifurcations of cycles. In CL_MATCONTM, LP, PD, and NS curves for period- $K$ cycles are computed by the mentioned Gauss-Newton continuation algorithm applied to minimally extended defining systems; cf. [13]. These systems were first implemented, together with the standard extended defining systems, in CONTENT [12]. We have adopted in CL_MATCONTM the most robust and efficient methods tested there. For completeness we include a brief description of the defining systems, as we will refer to them in section 4 and use some of the auxiliary variables for the detection of singularities.

The limit point curve and period-doubling curve are both defined by the following system:

$$
\left\{\begin{array}{cc}
g(x, \alpha)-x & =0 \\
s(x, \alpha) & =0
\end{array}\right.
$$

where $(x, \alpha) \in \mathbb{R}^{n+2}, g$ is given by (1.3), while $s$ is obtained by solving one of the algebraic systems

$$
\left(\begin{array}{cc}
g_{x}(x, \alpha) \mp I_{n} & w_{\text {bor }} \\
v_{\text {bor }}^{T} & 0
\end{array}\right)\left(\begin{array}{l}
v \\
s
\end{array}\right)=\left(\begin{array}{c}
0_{n} \\
1
\end{array}\right)
$$

where $w_{\text {bor }}, v_{\text {bor }} \in \mathbb{R}^{n}$ are chosen such that the matrix in (2.5) is nonsingular. One should take the "-" sign in (2.5) for the LP-curve and the "+" sign for the PD-curve. The derivatives of $s$ can be obtained easily from the derivatives of $g_{x}(x, \alpha)$ :

$$
s_{z}=-w^{T}\left(g_{x}\right)_{z} v,
$$

where $z$ is a state variable or an active parameter and $w$ is obtained by solving

$$
\left(\begin{array}{cc}
g_{x}^{T}(x, \alpha) \mp I_{n} & v_{b o r} \\
w_{b o r}^{T} & 0
\end{array}\right)\left(\begin{array}{c}
w \\
s
\end{array}\right)=\left(\begin{array}{c}
0_{n} \\
1
\end{array}\right) .
$$

We note that the quantities called $s$ in (2.5) and (2.7) are the same since they are both equal to the bottom-right element of the inverse of the square matrix in (2.5).

The NS and neutral-saddle curves are defined by the following system:

$$
\left\{\begin{aligned}
g(x, \alpha)-x & =0, \\
s_{i_{1} j_{1}}(x, \alpha, \kappa) & =0, \\
s_{i_{2} j_{2}}(x, \alpha, \kappa) & =0,
\end{aligned}\right.
$$


TABLE 2.2

Detection of codim 2 bifurcations of cycles.

\begin{tabular}{|r|c|c|c|}
\hline & LP & PD & NS \\
\hline \hline CP & $a_{1}=0$ & & \\
\hline GPD & & $b_{1}=0$ & $c_{1}=\Re\left(d_{1}\right)=0$ \\
\hline CH & & & $\operatorname{det}\left(A-I_{n}\right)=\kappa-1=0$ \\
\hline R1 & $\langle w, v\rangle=0$ & $\langle w, v\rangle=0$ & $\operatorname{det}\left(A+I_{n}\right)=\kappa+1=0$ \\
\hline R2 & & & $\kappa+\frac{1}{2}=0$ \\
\hline R3 & & $\operatorname{det}\left(A-I_{n}\right)=0$ & $\kappa=0$ \\
\hline R4 & & & $\operatorname{det}\left(A-I_{n}\right)=0, \kappa-1 \neq 0$ \\
\hline LPPD & $\operatorname{det}\left(A+I_{n}\right)=0$ & $\operatorname{det}\left(A \odot A-I_{m}\right)=0$ & $\operatorname{det}\left(A+I_{n}\right)=0, \kappa+1 \neq 0$ \\
\hline LPNS & $\operatorname{det}\left(A \odot A-I_{m}\right)=0$ & & $\operatorname{det}\left(A_{1} \odot A_{1}-I_{m_{1}}\right)=0$ \\
\hline PDNS & & & \\
\hline NSNS & & & \\
\hline \hline
\end{tabular}

TABLE 2.3

Smooth normal forms for generic codim 1 bifurcations of fixed points on center manifolds.

\begin{tabular}{|c|c|c|c|}
\hline & Eigenvectors & Normal form & Critical coefficients \\
\hline LP & $\begin{aligned} A q & =q \\
A^{T} p & =p\end{aligned}$ & $\begin{array}{l}w \mapsto \beta+w+a_{1} w^{2} \\
+O\left(w^{3}\right), \quad w \in \mathbb{R}\end{array}$ & $a_{1}=\frac{1}{2}\langle p, B(q, q)\rangle$ \\
\hline PD & $\begin{aligned} A q & =-q \\
A^{T} p & =-p\end{aligned}$ & $\begin{aligned} w & \mapsto-(1+\beta) w+b_{1} w^{3} \\
& +O\left(w^{4}\right), \quad w \in \mathbb{R}\end{aligned}$ & $\begin{array}{c}b_{1}=\frac{1}{6}\left\langle p, C(q, q, q)+3 B\left(q, h_{200}\right)\right\rangle \\
h_{200}=\left(I_{n}-A\right)^{-1} B(q, q)\end{array}$ \\
\hline NS & $\begin{array}{c}A q=\mathrm{e}^{i \theta_{0}} q \\
A^{T} p=\mathrm{e}^{-i \theta_{0}} p \\
\mathrm{e}^{i \nu \theta_{0}} \neq 1 \\
\nu=1,2,3,4\end{array}$ & $\begin{aligned} w \mapsto & w e^{i \theta}\left(1+\beta+d_{1}|w|^{2}\right) \\
& +O\left(|w|^{4}\right), \quad w \in \mathbb{C}\end{aligned}$ & $\begin{aligned} d_{1}= & \frac{1}{2} e^{-i \theta_{0}}\langle p, C(q, q, \bar{q}) \\
& +2 B\left(q, h_{1100}\right) \\
& \left.+B\left(\bar{q}, h_{2000}\right)\right\rangle \\
h_{1100}= & \left(I_{n}-A\right)^{-1} B(q, \bar{q}) \\
h_{2000}= & \left(e^{2 i \theta_{0}} I_{n}-A\right)^{-1} B(q, q)\end{aligned}$ \\
\hline
\end{tabular}

i.e. by $n+2$ equations for the $(n+3)$ unknowns $x \in \mathbb{R}^{n}, \alpha \in \mathbb{R}^{2}, \kappa \in \mathbb{R}$. Here $\left(i_{1}, j_{1}, i_{2}, j_{2}\right) \in\{1,2\}$ and $s_{i, j}$ are the components of $S$,

$$
S=\left(\begin{array}{ll}
s_{11} & s_{12} \\
s_{21} & s_{22}
\end{array}\right)
$$

which is obtained by solving

$$
\left(\begin{array}{cc}
\left(g_{x}\right)^{2}(x, \alpha)-2 \kappa g_{x}+I_{n} & W_{b o r} \\
V_{b o r}^{T} & O
\end{array}\right)\left(\begin{array}{c}
V \\
S
\end{array}\right)=\left(\begin{array}{c}
0_{n, 2} \\
I_{2}
\end{array}\right)
$$

where $V_{b o r}, W_{\text {bor }} \in \mathbb{R}^{n \times 2}$ are chosen (and can be adapted) so that the matrix in (2.9) is nonsingular. Along the NS curve, $\kappa$ is the real part of the critical multipliers $\mathrm{e}^{ \pm i \theta}$. The derivatives of $s_{i j}$ can be obtained easily from the derivatives of $g_{x}(x, \alpha)$ as before.

Table 2.2 specifies test functions used in CL_MATCONTM to detect and locate relevant codim 2 singularities along the codim 1 bifurcation curves. Here $a_{1}$ and $b_{1}$ are the critical normal form coefficients given in Table 2.3, where $q$ and $p$ should be replaced by the vectors $v$ and $w$, respectively, computed in (2.5) and (2.7). The test function $c_{1}$ is given by the Lyapunov coefficient $c_{1}=\Re\left(d_{1}\right)$, where $d_{1}$ is also specified in Table 2.3. Matrix $A_{1}$ is defined as $A_{1}=\left.A\right|_{N^{\perp}}$, where $A=g_{x}$ and $N^{\perp}$ is the orthogonal complement in $\mathbb{R}^{n}$ of the two-dimensional eigenspace associated with the pair of multipliers with unit product of $A^{T} ; m_{1}=\frac{1}{2}(n-2)(n-3)$.

It is possible and sometimes necessary to adapt the defining system while continuing a bifurcation curve, i.e., to update the auxiliary variables used in the defining 
system of the computed branch. The bordering vectors $v_{b o r}$ and $w_{b o r}$ may require updating since they must ensure that the matrices in (2.5) and (2.7) are nonsingular. Updating is done in CL_MATCONTM by replacing $v_{b o r}$ and $w_{\text {bor }}$ with the normalized vectors $v, w$ computed in (2.5), (2.7), respectively. Updating of $V$ and $W$ in (2.9) is done similarly, while $\left(i_{1}, j_{1}, i_{2}, j_{2}\right)$ are updated in such a way that the linearized system of (2.8) is as well-conditioned as possible.

\section{Numerical analysis of codim 2 bifurcations.}

3.1. Codim 2 cases. While, as mentioned in the introduction, methods to study codim 1 bifurcations are implemented in several packages [12], the numerical analysis of codim 2 bifurcations has remained a laborious task. We implemented in CL_MATCONTM the formulas for the critical center manifold reduction from $[19,20]$ for all eleven codim 2 bifurcations. Below we give normal forms to which the restriction of a generic map $g(x, \alpha)=f^{(K)}(x, \alpha)$ to the parameter-dependent center manifold can be transformed near the corresponding bifurcation by smooth invertible coordinate and parameter transformations. When CL_MATCONTM detects a codim 2 bifurcation, the critical coefficients of these normal forms are automatically computed and reported to the user, so that nondegeneracy is now easily checked. We stress that they can also be used to predict certain behavior of the system. In 6 out of 11 cases, branches of local codim 1 bifurcations are rooted at codim 2 bifurcations, and for these cases we also incorporate the parameter-dependent part of the normal form and provide asymptotic expressions for the new curves. In section 3.2 we specify how we switch the continuation to those branches.

The $\mathcal{O}$-symbol denotes higher order terms in phase-variables, the coefficients of which may also depend on parameters. But the qualitative picture is determined by the lowest order terms listed below. We refer to [18, Chapter 9] and $[19,20]$ for more details, including explicit expressions for all critical normal form coefficients which are not repeated in the present paper. If a complex critical eigenvalue $\lambda$ is involved, it is always assumed that $\lambda^{\nu} \neq 1$ for $\nu=1,2,3,4$. In some cases, we combine two real unfolding parameters $\left(\beta_{1}, \beta_{2}\right)$ into one complex parameter $\beta=\beta_{1}+i \beta_{2} \in \mathbb{C}$.

3.1.1. CP. The critical smooth normal form on the center manifold at a cusp bifurcation is

$$
w \mapsto w+a_{2} w^{3}+\mathcal{O}\left(|w|^{4}\right), \quad w \in \mathbb{R},
$$

where, generically, $a_{2} \neq 0$. Under this condition, a generic two-parameter unfolding of this singularity has two fold curves in the parameter plane which form a cuspidal wedge. For nearby parameter values, the map $g$ has up to three fixed points that pairwise collide along the fold curves. In the direct product of the state and the parameter spaces, there is one smooth fold curve, so no branch switching is needed. CL_MATCONTM reports the value of $a_{2}$ at the bifurcation.

3.1.2. GPD. Near a generalized flip bifurcation the restriction of the map $g$ to the parameter-dependent center manifold is smoothly equivalent to the normal form

$$
w \mapsto-\left(1+\beta_{1}\right) w+\beta_{2} w^{3}+b_{2}(\beta) w^{5}+\mathcal{O}\left(|w|^{6}\right), \quad w \in \mathbb{R},
$$

where, generically, the coefficient $b_{2}(0) \neq 0$, while the components of $\beta=\left(\beta_{1}, \beta_{2}\right)$ are smooth functions of $\alpha$, which can serve as new unfolding parameters. The value of $b_{2}(0)$ is reported by the software. The fixed point $w=0$ of the map (3.2) exhibits a flip bifurcation for $\beta_{1}=0$. It is well known that from the point $\beta=0$, corresponding 
to the degenerate flip bifurcation, a fold curve of double-period cycles emanates. The asymptotic expression for this curve in (3.2) is given by

$$
\left(w, \beta_{1}, \beta_{2}\right)=\left(\varepsilon,-b_{2} \varepsilon^{4}+\mathcal{O}\left(\varepsilon^{5}\right),-2 b_{2} \varepsilon^{2}+\mathcal{O}\left(\varepsilon^{3}\right)\right) .
$$

3.1.3. CH. If $\mathrm{e}^{i \nu \theta_{0}} \neq 1$ for $\nu=1,2, \ldots, 6$, the critical smooth normal form on the center manifold at the Chenciner bifurcation can be written as

$$
z \mapsto z \mathrm{e}^{i \theta_{0}}\left(1+d_{1}|z|^{2}+d_{2}|z|^{4}+\mathcal{O}\left(|z|^{6}\right)\right), \quad z \in \mathbb{C},
$$

where $c_{1}=\Re\left(d_{1}\right)=0$ but, generically, $c_{2}=\Re\left(d_{2}\right)+\frac{1}{2} \Im\left(d_{1}\right)^{2} \neq 0$ and is reported to the user. A generic two-parameter unfolding of this singularity has a complicated bifurcation set due to the "collision" and destruction of two closed invariant curves of different stability born via the sub- and supercritical NS bifurcations, respectively. There are no cycle bifurcation curves rooted at this bifurcation.

3.1.4. R1. The restriction of the map at a $1: 1$ resonance to the corresponding center manifold can be written in the form

$$
\left(\begin{array}{c}
w_{1} \\
w_{2}
\end{array}\right) \mapsto\left(\begin{array}{c}
w_{1}+w_{2} \\
w_{2}+a_{1} w_{1}^{2}+b_{1} w_{1} w_{2}
\end{array}\right)+\mathcal{O}\left(\|w\|^{3}\right), \quad w \in \mathbb{R}^{2} .
$$

Generically, an NS bifurcation curve of the fixed point meets the fold bifurcation curve tangentially. CL_MATCONTM reports the sign of $a_{1}\left(b_{1}-2 a_{1}\right)$, giving the first Lyapunov coefficient along the NS curve near the bifurcation. The local branch switching problem is trivial here, since both curves correspond to fixed points of $g$. The full bifurcation diagram near the codim 2 point is complicated and involves global bifurcations, e.g., tangencies of stable and unstable invariant manifolds of saddle fixed points of $g$ and destruction of a closed invariant curve born via the NS bifurcation.

3.1.5. R2. Near a 1:2 resonance the restriction of the map $g$ to the parameterdependent center manifold is smoothly equivalent to the normal form

$$
\begin{aligned}
\left(\begin{array}{c}
w_{1} \\
w_{2}
\end{array}\right) & \mapsto\left(\begin{array}{c}
-w_{1}+w_{2} \\
\beta_{1} w_{1}+\left(-1+\beta_{2}\right) w_{2}+C_{1}(\beta) w_{1}^{3}+D_{1}(\beta) w_{1}^{2} w_{2}
\end{array}\right) \\
& +\mathcal{O}\left(\|w\|^{4}\right), \quad w \in \mathbb{R}^{2}
\end{aligned}
$$

which depends on two unfolding parameters $\left(\beta_{1}, \beta_{2}\right)$. If $C_{1}(0)<0$, then there is an NS curve of fixed points of $g$ with double period that emanates from the flip bifurcation curve $\beta_{2}=0$ of fixed points. It has the following asymptotic expression:

$$
\left(w_{1}^{2}, w_{2}, \beta_{1}, \beta_{2}\right)=\left(-\frac{1}{C_{1}}, 0,1,\left(2+\frac{D_{1}}{C_{1}}\right)\right) \varepsilon+\mathcal{O}\left(\varepsilon^{2}\right) .
$$

There are also global bifurcations associated with the destruction of closed invariant curves. CL_MATCONTM reports the values of $4 C_{1}(0)$ and $-2 D_{1}(0)-6 C_{1}(0)$ (relevant for the flow approximation) to the user.

3.1.6. R3. At a $1: 3$ resonance the restriction of the map $g$ to the parameterdependent center manifold is smoothly equivalent to the normal form

$$
z \mapsto\left(\mathrm{e}^{2 i \pi / 3}+\beta\right) z+B_{1}(\beta) \bar{z}^{2}+C_{1}(\beta) z|z|^{2}+\mathcal{O}\left(|z|^{4}\right), \quad z \in \mathbb{C},
$$


where $\beta=\beta_{1}+i \beta_{2} \in \mathbb{C}$. A generic unfolding of this singularity has a period-3 saddle cycle that does not bifurcate for nearby parameter values, although it merges with the primary fixed point as the parameters approach R3. Only global bifurcations related to the destruction of a closed invariant curve born via the primary NS bifurcation occur in a neighborhood of this codim 2 point.

Note that the period-3 cycle becomes neutral near this bifurcation. Recall that a saddle cycle is called neutral if the corresponding fixed point has a pair of real eigenvalues with product 1 . This singularity is important in analyzing global bifurcations of invariant manifolds of cycles. Moreover, the curve of neutral period-3 saddle cycles may turn into a true NS bifurcation at R1 or R2. Therefore, we give here an asymptotic of this curve.

First we need a vector field for which the time- 1 flow approximates the third iterate of the map, i.e.,

$$
\widetilde{g}(\eta, \widetilde{\beta})=\widetilde{\beta} \eta+\bar{\eta}^{2}+C_{0}(\beta) \eta^{2} \bar{\eta}+\mathcal{O}\left(|\eta|^{4}\right),
$$

where

$$
\widetilde{\beta}=3 \mathrm{e}^{-2 i \pi / 3} \beta, \quad z=\frac{1}{\left|B_{1}(\beta)\right|} \mathrm{e}^{i \arg \left(B_{1}(\beta)\right) / 3} \eta, \quad C_{0}(\beta)=\frac{1}{3}\left(\frac{C_{1}(\beta)}{\left|B_{1}(\beta)\right|^{2}} \mathrm{e}^{-2 i \pi / 3}-1\right) .
$$

We write $C_{0}=a+i b$, where $a$ is reported upon detection, so that for $\eta=\rho \mathrm{e}^{i \phi}$ the neutral saddle curve has the following asymptotic expression:

$$
\left(\rho, \phi, \beta_{1}, \beta_{2}\right)=\left(\varepsilon, s(\pi / 6-a \varepsilon / 3),-2 a \varepsilon^{2}, s \varepsilon-b \varepsilon^{2}\right)+\mathcal{O}\left(\varepsilon^{3}\right),
$$

where $s= \pm 1$.

3.1.7. R4. Near a 1:4 resonance the restriction of the map $g$ to the parameterdependent center manifold is smoothly equivalent to the normal form

$$
z \mapsto(i+\beta) z+C_{1}(\beta) z^{2} \bar{z}+D_{1}(\beta) \bar{z}^{3}+\mathcal{O}\left(|z|^{4}\right), \quad z \in \mathbb{C},
$$

where $\beta=\beta_{1}+i \beta_{2} \in \mathbb{C}$. For this bifurcation we need not only this parameterdependent normal form, but also an approximation of its fourth iterate by a unit-time shift along orbits of a vector field

$$
\widetilde{g}(\eta, \widetilde{\beta})=\widetilde{\beta} \eta+A_{0}(\beta) \eta^{2} \bar{\eta}+\bar{\eta}^{3}+\mathcal{O}\left(|\eta|^{4}\right),
$$

where $\eta \in \mathbb{C}$ and $\widetilde{\beta}=\tilde{\beta}_{1}+i \tilde{\beta}_{2}, \tilde{\beta}_{i} \in \mathbb{R}$. Here we use

$$
z=\frac{1}{\sqrt{\left|D_{1}(\beta)\right|}} \mathrm{e}^{i \arg \left(D_{1}(\beta)\right) / 4} \eta, \quad A_{0}(\beta)=-i \frac{C_{1}(\beta)}{\left|D_{1}(\beta)\right|} .
$$

Moreover, we have

$$
\left(\begin{array}{l}
\widetilde{\beta}_{1} \\
\widetilde{\beta}_{2}
\end{array}\right)=\left(\begin{array}{cc}
0 & 4 \\
-4 & 0
\end{array}\right)\left(\begin{array}{l}
\beta_{1} \\
\beta_{2}
\end{array}\right) .
$$

There are three possible branch switches for this bifurcation. Denote the reported values by $a=\Re\left(A_{0}(0)\right)$ and $b=\Im\left(A_{0}(0)\right)$. If $\Delta \equiv a^{2}+b^{2}-1>0$, then there are two half-lines $l_{1,2}$ of a limit-point curve of cycles with four times the original period. If

$$
|b|>\frac{\left(1+a^{2}\right)}{\sqrt{1-a^{2}}},
$$

Copyright $@$ by SIAM. Unauthorized reproduction of this article is prohibited. 
then there is a curve $n_{1}$ along which a cycle of four times the primary period exhibits an NS bifurcation. Using $\eta=r e^{i \phi}$, we have the following approximations:

$$
\begin{gathered}
l_{1,2}:\left(r^{2}, \phi, \widetilde{\beta}_{1}, \widetilde{\beta}_{2}\right)=\left(\varepsilon, \frac{1}{4} \arctan \left(\frac{a b \pm \sqrt{\Delta}}{b^{2}-1}\right)+\mathcal{O}(\varepsilon),\right. \\
\left.\frac{-a \Delta \mp b \sqrt{\Delta}}{a^{2}+b^{2}} \varepsilon, \frac{-b \Delta \pm a \sqrt{\Delta}}{a^{2}+b^{2}} \varepsilon\right)+\mathcal{O}\left(\varepsilon^{2}\right), \\
n_{1}:\left(r^{2}, \phi, \widetilde{\beta}_{1}, \widetilde{\beta}_{2}\right)=\left(\varepsilon+\mathcal{O}\left(\varepsilon^{2}\right), \operatorname{sign}(b) \arccos (a) / 4+\mathcal{O}(\varepsilon),\right. \\
\left.-2 a \varepsilon+\mathcal{O}\left(\varepsilon^{2}\right),-\left(b-\operatorname{sign}(b) \sqrt{1-a^{2}}\right) \varepsilon+\mathcal{O}\left(\varepsilon^{2}\right)\right) .
\end{gathered}
$$

Taking into account (3.13), we obtain expressions for the unfolding parameters $\beta_{1}$ and $\beta_{2}$. If, in the formula for $n_{1}$, we replace $\operatorname{sign}(b)$ by $-\operatorname{sign}(b)$, then this gives the asymptotic for a neutral saddle singularity of the period- 4 cycle.

Generically, there are also global bifurcations near R4.

3.1.8. LPPD. Near a fold-flip bifurcation, the restriction of the map $g$ to the parameter-dependent center manifold is smoothly equivalent to the normal form

$$
\begin{aligned}
\left(\begin{array}{l}
w_{1} \\
w_{2}
\end{array}\right) & \mapsto\left(\begin{array}{c}
\beta_{1}+\left(1+\beta_{2}\right) w_{1}+a(\beta) w_{1}^{2}+b(\beta) w_{2}^{2}+c_{1}(\beta) w_{1}^{3}+c_{2}(\beta) w_{1} w_{2}^{2} \\
-w_{2}+e(\beta) w_{1} w_{2}+c_{3}(\beta) w_{1}^{2} w_{2}+c_{4}(\beta) w_{2}^{3}
\end{array}\right) \\
& +\mathcal{O}\left(\|w\|^{4}\right), \quad w \in \mathbb{R}^{2}
\end{aligned}
$$

A new branch predicted by (3.15) for a generic map $g$ is an NS bifurcation of double period that exists if $b e>0$ and has the asymptotic expression

$$
\left(x, y^{2}, \beta_{1}, \beta_{2}\right)=\left(-\frac{c_{4}}{e}, 1,-b,-\frac{2 b+e c_{2}-2(a+e) c_{4}}{e}\right) \varepsilon+\mathcal{O}\left(\varepsilon^{2}\right) .
$$

CL_MATCONTM reports the coefficients $\frac{a}{e}$ and $b e$ and the sign of the Lyapunov coefficient if applicable. As for the majority of the considered cases, there are also global bifurcations near this codim 2 point.

3.1.9. LPNS. For a fold-NS bifurcation, the critical normal form on the center manifold is given by

$$
\left(\begin{array}{c}
w \\
z
\end{array}\right) \mapsto\left(\begin{array}{c}
w+s z \bar{z}+w^{2}+c x^{3} \\
e^{i \theta_{0}} z+a w z+b z w^{2}
\end{array}\right)+\mathcal{O}\left(\|(w, z)\|^{4}\right), \quad(w, z) \in \mathbb{R} \times \mathbb{C} .
$$

The critical coefficients $s, a, b, c$ are reported. Depending on their values, several bifurcation scenarios are possible in parameter-dependent unfoldings, which all involve global phenomena.

3.1.10. PDNS. Near a flip-NS bifurcation, the restriction of the map $g$ to the parameter-dependent center manifold is smoothly equivalent to the parameterdependent normal form

$$
\begin{gathered}
\left(\begin{array}{c}
w \\
z
\end{array}\right) \mapsto\left(\begin{array}{c}
-w\left(1+\beta_{1}+c_{1}(\beta) w^{2}+c_{2}(\beta)|z|^{2}\right) \\
z e^{i \theta(\beta)}\left(1+\beta_{2}+c_{3}(\beta) w^{2}+c_{4}(\beta)|z|^{2}\right)
\end{array}\right)+\mathcal{O}\left(\|(w, z)\|^{4}\right), \\
(w, z) \in \mathbb{R} \times \mathbb{C}
\end{gathered}
$$

Copyright (C) by SIAM. Unauthorized reproduction of this article is prohibited. 
where $\Re\left(c_{i}(0)\right)$ are reported and $\theta(0)=\theta_{0}$. Besides global bifurcations, an NS bifurcation curve of double period for $g$ is rooted at $\beta=0$; it is always present. The asymptotic expression of this curve is given by

$$
\left(w^{2}, z, \beta_{1}, \beta_{2}\right)=\left(1,0,-c_{1},-\operatorname{sign}\left(c_{1}\right) \Re\left(c_{3}\right)\right) \varepsilon+\mathcal{O}\left(\varepsilon^{2}\right) .
$$

3.1.11. NSNS. For a double NS bifurcation, provided that $l \theta_{0} \neq j \theta_{1}$ for integer $l$ and $j$ with $l+j \leq 4$, the critical normal form on the center manifold is

$$
\left(\begin{array}{c}
z_{1} \\
z_{2}
\end{array}\right) \mapsto\left(\begin{array}{l}
z_{1}\left(e^{i \theta_{0}}+c_{1}\left|z_{1}\right|^{2}+c_{2}\left|z_{2}\right|^{2}\right) \\
z_{2}\left(e^{i \theta_{1}}+c_{3}\left|z_{1}\right|^{2}+c_{4}\left|z_{2}\right|^{2}\right)
\end{array}\right)+\mathcal{O}\left(\|z\|^{4}\right), \quad z \in \mathbb{C}^{2} .
$$

Depending on the values of $\Re\left(c_{i}\right)$, which are reported, several bifurcation scenarios are possible in parameter-dependent unfoldings, which all involve global phenomena. To analyze some of them, one has to take into account fourth- and fifth-order terms.

3.2. Branch switching from codim 2 bifurcations. Here we address the problem of branch switching at codim 2 bifurcation points of maps, when the emanating curve corresponds to a local bifurcation. This involves the degenerate flip, 1:2 resonance, 1:4 resonance, fold-flip, and flip-NS bifurcations only. To obtain appropriate initial continuation data for the original map, we combine parameter-dependent center-manifold reduction with asymptotic expressions for the new curves given in section 3.1.

Although we know that in several cases global bifurcations are also involved, we will not try to switch to those branches, as the continuation of these global bifurcations is not supported by the current version of CL_MATCONTM.

3.2.1. Parameter-dependent center-manifold reduction. In all our cases, the map $g(x, \alpha): \mathbb{R}^{n} \times \mathbb{R}^{2} \rightarrow \mathbb{R}^{n}$, where $g$ is defined by (1.3), satisfies $g\left(x_{0}, \alpha_{0}\right)=x_{0}$, and its Jacobian matrix $A=g_{x}\left(x_{0}, \alpha_{0}\right)$ has at most three multipliers on the unit circle. Furthermore we know a parameter-dependent smooth normal form $G(w, \beta)$ for the corresponding bifurcation; see section 3.1. Then we assume a relation

$$
\alpha-\alpha_{0}=V(\beta)=v_{10} \beta_{1}+v_{01} \beta_{2}+\mathcal{O}\left(\|\beta\|^{2}\right)
$$

between the original and the unfolding parameters. Note that $V$ incorporates linear scalings. Occasionally, we interpret $\beta_{1}=\bar{\beta}_{2}$ as one parameter $\beta \in \mathbb{C}$; in such cases, $v_{01}=\bar{v}_{10} \in \mathbb{C}^{2}$.

To find a parameter-dependent center manifold as the graph of $x=x_{0}+H(w, \beta)$ we make a Taylor expansion of the homological equation

$$
g\left(x_{0}+H(w, \beta), \alpha_{0}+V(\beta)\right)=H(G(w, \beta), \beta)
$$

in $w$ and $\beta$ at $(w, \beta)=(0,0)$, where we expand $g$ as in (2.2) and write

$$
H(w, \beta)=\sum_{|\mu|+|\nu| \geq 1} h_{\mu, \nu} w^{\mu} \beta^{\nu}
$$

and $\mu, \nu$ are multi-indices. All coefficients must vanish, and this leads to a solution for $H$ and $V$. Below we will focus only on the parameter-dependent computations and assume full knowledge of the critical center manifold and the critical normal form coefficients; see $[18,19,20]$. The solvability conditions imposed coincide with the transversality of the original family to the bifurcation manifold. A similar technique 
was introduced in [5, section 11] to switch at codim 2 bifurcations of equilibria in ODEs.

Initial data for the new curve is now provided by substituting for $w$ and $\beta$ the asymptotic expression in $\varepsilon$ of section 3.1 into $H$ and $V$ up to a certain order in $\varepsilon$, usually 2. In CL_MATCONTM the user can specify $\varepsilon$ to obtain a starting point near the emanating curve for which the continuation code converges to a point on the new curve.

It will be convenient to introduce some notation. Let $p$ denote an eigenvector of $A^{T}$ corresponding to the eigenvalue -1 of $A$. We will then write $\Gamma: \mathbb{R}^{n+2} \rightarrow \mathbb{R}^{n}$ for $\Gamma(q, v)=\left\langle p, A_{1}(q, v)+B\left(q,\left(I_{n}-A\right)^{-1} J_{1} v\right)\right\rangle$ and $\gamma_{i}=\Gamma\left(q, e_{i}\right)$ for the evaluation of $\Gamma$ on the standard basis vectors in $\mathbb{R}^{2}$. If $\gamma_{i} \neq 0$ for $i=1,2$, then $s_{1}=\frac{1}{\left(\gamma_{1}^{2}+\gamma_{2}^{2}\right)}\left(\gamma_{1}, \gamma_{2}\right)^{T}$ and $s_{2}=\left(-\gamma_{2}, \gamma_{1}\right)^{T}$ compose a new orthogonal basis in $\mathbb{R}^{2}$.

3.2.2. Degenerate flip. The homological equation (3.22) provides the following systems to be solved:

$$
\begin{aligned}
& \left(A-I_{n}\right)\left[h_{010}, h_{001}\right]=-J_{1}\left[v_{10}, v_{01}\right], \\
& \left(A+I_{n}\right)\left[h_{110}, h_{101}\right]=-[q, 0]-A_{1}\left(q,\left[v_{10}, v_{01}\right]\right)-B\left(q,\left[h_{010}, h_{001}\right]\right),
\end{aligned}
$$

where $[a, b]$ is an $n \times 2$-matrix with columns $a, b \in \mathbb{R}^{n}$. The higher orders give

$$
\begin{gathered}
\left(A-I_{n}\right) h_{210}=2 h_{200}-\left[B_{1}\left(q, q, v_{10}\right)+B\left(h_{200}, h_{010}\right)+A_{1}\left(h_{200}, v_{10}\right)\right. \\
\left.+2 B\left(q, h_{110}\right)+C\left(q, q, h_{010}\right)\right] \\
\left(A-I_{n}\right) h_{201}=-\left[B_{1}\left(q, q, v_{01}\right)+B\left(h_{200}, h_{001}\right)+A_{1}\left(h_{200}, v_{01}\right)\right. \\
\left.+2 B\left(q, h_{101}\right)+C\left(q, q, h_{001}\right)\right] \\
\left(A+I_{n}\right) h_{310}=-3 h_{300}-\left[D\left(q, q, q, h_{010}\right)+3 C\left(q, q, h_{110}\right)+C_{1}\left(q, q, q, v_{10}\right)\right. \\
+3 B\left(h_{110}, h_{200}\right)+B\left(h_{300}, h_{010}\right)+3 B_{1}\left(h_{200}, q, v_{10}\right) \\
\left.+A_{1}\left(h_{300}, v_{10}\right)+3 C\left(h_{200}, q, h_{010}\right)+3 B\left(h_{210}, q\right)\right] \\
\left(A+I_{n}\right) h_{301}=6 q-[ \\
+\left(q, q, q, h_{001}\right)+3 C\left(q, q, h_{101}\right)+C_{1}\left(q, q, q, v_{01}\right) \\
+3 B\left(h_{101}, h_{200}\right)+B\left(h_{300}, h_{001}\right)+3 B_{1}\left(h_{200}, q, v_{01}\right) \\
\left.+A_{1}\left(h_{300}, v_{01}\right)+3 C\left(h_{200}, q, h_{001}\right)+3 B\left(h_{201}, q\right)\right]
\end{gathered}
$$

The linear part of $V$ involves four unknowns to be found from equations with singular left-hand sides, i.e., (3.25), (3.28), (3.29). For the solution we start with substitution of $\left[h_{010}, h_{001}\right]=\left(I_{n}-A\right)^{-1} J_{1}\left[v_{10}, v_{01}\right]$ from (3.24) into (3.25). As the left-hand side of (3.25) is singular, the right-hand side must be orthogonal to the adjoint eigenvector $p$. Application of the Fredholm alternative leads to

$$
\begin{aligned}
& \left\langle p,\left(A+I_{n}\right)\left[h_{110}, h_{101}\right]\right\rangle \\
& \quad=-\left\langle p,[q, 0]+A_{1}\left(q,\left[v_{10}, v_{01}\right]\right)+B\left(q,\left(I_{n}-A\right)^{-1} J_{1}\left[v_{10}, v_{01}\right]\right)\right\rangle .
\end{aligned}
$$

We see that the operator $\Gamma(q, v)$ appears naturally and rewrite it as

$$
\left[\gamma_{1}, \gamma_{2}\right]\left[v_{10}, v_{01}\right]=[-1,0] .
$$

Copyright $@$ by SIAM. Unauthorized reproduction of this article is prohibited. 
The general solution is given by

$$
v_{10}=-s_{1}+\delta_{1} s_{2}, \quad v_{01}=\delta_{2} s_{2}, \quad \delta_{1}, \delta_{2} \in \mathbb{R}
$$

Since $v_{10}, v_{01}$ appear linearly in these equations (via the multilinear functions), application of the Fredholm alternative to (3.28), (3.29) results in a linear system for the constants $\delta_{1}, \delta_{2}$.

3.2.3. 1:2 resonance. As before, we first list the necessary equations obtained from the homological equation

$$
\begin{aligned}
\left(A-I_{n}\right)\left[h_{0010}, h_{0001}\right]= & -J_{1}\left[v_{10}, v_{01}\right] \\
\left(A+I_{n}\right)\left[h_{1010}, h_{1001}\right]= & {\left[q_{1}, 0\right]-A_{1}\left(q_{0},\left[v_{10}, v_{01}\right]\right)-B\left(q_{0},\left[h_{0010}, h_{0001}\right]\right) } \\
\left(A+I_{n}\right)\left[h_{0110}, h_{0101}\right]= & {\left[h_{1010}, q_{1}+h_{1001}\right]-A_{1}\left(q_{1},\left[v_{10}, v_{01}\right]\right) } \\
& -B\left(q_{1},\left[h_{0010}, h_{0001}\right]\right)
\end{aligned}
$$

As for the degenerate flip, we substitute $\left[h_{0010}, h_{0001}\right]=\left(I_{n}-A\right)^{-1} J_{1}\left[v_{10}, v_{01}\right]$ into (3.31). As (3.31) is similar to (3.25), the solution for $v_{10}$ and $v_{01}$ is now

$$
v_{10}=s_{1}+\delta_{1} s_{2}, \quad v_{01}=\delta_{2} s_{2}, \quad \delta_{1}, \delta_{2} \in \mathbb{R} .
$$

We substitute the $s_{i}$ into (3.32) and write

$$
\begin{array}{ll}
Q_{1}=\left\langle p_{1}, A_{1}\left(q_{0}, s_{1}\right)+B\left(q_{0},\left(A-I_{n}\right)^{-1} J_{1} s_{1}\right)\right\rangle, & Q_{2}=\Gamma\left(q_{1}, s_{1}\right), \\
Q_{3}=\left\langle p_{1}, A_{1}\left(q_{0}, s_{2}\right)+B\left(q_{0},\left(A-I_{n}\right)^{-1} J_{1} s_{2}\right)\right\rangle, & Q_{4}=\Gamma\left(q_{1}, s_{2}\right) .
\end{array}
$$

A little algebra shows that

$$
\delta_{1}=-\left(\frac{Q_{1}+Q_{2}}{Q_{3}+Q_{4}}\right), \quad \delta_{2}=\frac{1}{Q_{3}+Q_{4}} .
$$

3.2.4. 1:3 resonance. We follow a slightly different procedure here. We want to find $V(\beta)=v \beta+\bar{v} \bar{\beta}$, where $\beta=\beta_{1}+i \beta_{2}$. Then we treat $\beta$ and $\bar{\beta}$ as independent variables, which makes it slightly easier to find the solutions. As the final $V(\beta)$ should be real, it follows that $v=v_{10}=\bar{v}_{01}$.

Let $\lambda=\mathrm{e}^{2 i \pi / 3}$, and introduce $A q=\lambda q, A^{T} p=\bar{\lambda} p,\langle p, q\rangle=1$. As before, the first linear systems resulting from (3.22) are given by

$$
\begin{aligned}
\left(A-I_{n}\right)\left[h_{0010}, h_{0001}\right] & =-J_{1}\left[v_{10}, v_{01}\right] \\
\left(A-\lambda I_{n}\right)\left[h_{1010}, h_{1001}\right] & =[q, 0]-A_{1}\left(q,\left[v_{10}, v_{01}\right]\right)-B\left(q,\left[h_{0010}, h_{0001}\right]\right),
\end{aligned}
$$

and two complex conjugated systems for $h_{0101}$ and $h_{0110}$. With the same approach we will now find complex $\gamma_{i}$, and rewriting the system for $v=v_{10}=\bar{v}_{01}$, we have $\left(\gamma_{1}, \gamma_{2}\right) v=1,\left(\gamma_{1}, \gamma_{2}\right) \bar{v}=0$, with $v=\left(\bar{\gamma}_{2},-\bar{\gamma}_{1}\right) /\left(\gamma_{1} \bar{\gamma}_{2}-\gamma_{2} \bar{\gamma}_{1}\right)$ as solution.

3.2.5. 1:4 resonance. Replacing $\lambda=i$, we can repeat the procedure for the case of $1: 3$ resonance.

Copyright $@$ by SIAM. Unauthorized reproduction of this article is prohibited. 
3.2.6. Fold-flip. Let $A q_{1,2}= \pm q_{1,2}, A^{T} p_{1,2}= \pm p_{1,2},\left\langle p_{1}, q_{1}\right\rangle=\left\langle p_{2}, q_{2}\right\rangle=1$. The necessary systems to solve from the homological equation (3.22) are

$$
\begin{aligned}
\left(A-I_{n}\right)\left[h_{0010}, h_{0001}\right]= & {\left[q_{1}, 0\right]-J_{1}\left[v_{10}, v_{01}\right], } \\
\left(A-I_{n}\right)\left[h_{1010}, h_{1001}\right]= & {\left[h_{2000}, q_{1}\right]-A_{1}\left(q_{1},\left[v_{10}, v_{01}\right]\right) } \\
& -B\left(q_{1},\left[h_{0010}, h_{0001}\right]\right), \\
\left(A+I_{n}\right)\left[h_{0110}, h_{0101}\right]= & {\left[h_{1100}, 0\right]-A_{1}\left(q_{2},\left[v_{10}, v_{01}\right]\right) } \\
& -B\left(q_{2},\left[h_{0010}, h_{0001}\right]\right) .
\end{aligned}
$$

First note that all matrices in the left-hand sides are singular. If we take $\left(\gamma_{1}, \gamma_{2}\right)=$ $p_{1}^{T} J_{1}$, we can define the orthogonal vectors $s_{1}$ and $s_{2}$ as before, and then $v_{10}=s_{1}+\delta_{1} s_{2}$ and $v_{01}=\delta_{2} s_{2}$ solve system (3.33) for arbitrary $\delta_{1}, \delta_{2}$. Bordering the singular matrix $\left(A-I_{n}\right)$, one can solve for $h_{0010}$ and $h_{0001}$. Any multiple of $q_{1}$ can be added to $h_{0010}$ and $h_{0001}$, so we use $h_{0010}=\left(A-I_{n}\right)^{I N V}\left(q_{1}-J_{1} v_{10}\right)+\delta_{3} q_{1}$ and $h_{0010}=$ $-\left(A-I_{n}\right)^{I N V}\left(J_{1} v_{01}\right)+\delta_{4} q_{1}$. We will use this freedom to solve (3.34) and (3.35) simultaneously for all $\delta$ 's. Note that $h_{2000}$ and $h_{1100}$ are also found using bordered systems chosen, but such that $\left\langle p_{1}, h_{2000}\right\rangle=\left\langle p_{2}, h_{1100}\right\rangle=0$.

Then we obtain the following four-dimensional system:

$$
\left(\begin{array}{cc}
L & 0_{2 \times 2} \\
0_{2 \times 2} & L
\end{array}\right)\left(\begin{array}{l}
\delta_{1} \\
\delta_{3} \\
\delta_{2} \\
\delta_{4}
\end{array}\right)=\left(\begin{array}{c}
-\left\langle p_{1}, A_{1}\left(q_{1}, s_{1}\right)+B\left(q_{1},\left(A-I_{n}\right)^{I N V}\left(q_{1}-J_{1} s_{1}\right)\right)\right\rangle \\
-\left\langle p_{2}, A_{1}\left(q_{2}, s_{1}\right)+B\left(q_{2},\left(A-I_{n}\right)^{I N V}\left(q_{1}-J_{1} s_{1}\right)\right)\right\rangle \\
1 \\
0
\end{array}\right)
$$

where $L$ is defined by

$$
L=\left(\begin{array}{ll}
\left\langle p_{1}, A_{1}\left(q_{1}, s_{2}\right)+B\left(q_{1},\left(I_{n}-A\right)^{I N V} J_{1} s_{2}\right)\right\rangle & \left\langle p_{1}, B\left(q_{1}, q_{1}\right)\right\rangle \\
\left\langle p_{2}, A_{1}\left(q_{2}, s_{2}\right)+B\left(q_{2},\left(I_{n}-A\right)^{I N V} J_{1} s_{2}\right)\right\rangle & \left\langle p_{2}, B\left(q_{1}, q_{2}\right)\right\rangle
\end{array}\right) .
$$

Notice that $2 a(0)=\left\langle p_{1}, B\left(q_{1}, q_{1}\right)\right\rangle$ and that $q_{1}$ can be chosen such that $e(0)=$ $\left\langle p_{2}, B\left(q_{1}, q_{2}\right)\right\rangle=1$. The condition $\gamma_{1} \gamma_{2} \operatorname{det}(L) \neq 0$ is equivalent with the transversality to the bifurcation manifold of the family $g(x, \alpha)$.

3.2.7. Flip-NS. Introduce $A q_{1}=q_{1}, A^{T} p_{1}=p_{1},\left\langle p_{1}, q_{1}\right\rangle=1$ and $A q_{2}=$ $e^{i \theta_{0}} q_{2}, A^{T} p_{2}=e^{-i \theta_{0}} p_{2},\left\langle p_{2}, q_{2}\right\rangle=1$. The linear systems obtained from the homological equation (3.22) are

$$
\begin{aligned}
\left(A-I_{n}\right)\left[h_{00010}, h_{00001}\right] & =-J_{1}\left[v_{10}, v_{01}\right], \\
\left(A+I_{n}\right)\left[h_{10010}, h_{10001}\right] & =\left[-q_{1}, 0\right]-A_{1}\left(q_{1},\left[v_{10}, v_{01}\right]\right)-B\left(q_{1},\left[h_{00010}, h_{00001}\right]\right), \\
\left(A-e^{i \theta_{0}} I_{n}\right)\left[h_{01010}, h_{01001}\right] & =\left[0, q_{2} e^{i \theta_{0}}\right]-A_{1}\left(q_{2},\left[v_{10}, v_{01}\right]\right)-B\left(q_{2},\left[h_{00010}, h_{00001}\right]\right) .
\end{aligned}
$$

The same approach as for the degenerate flip and 1:2-resonance cases is to substitute the formal solution of the first equation into the second, and we write

$$
v_{10}=-s_{1}+\delta_{1} s_{2}, \quad v_{01}=\delta_{2} s_{2},
$$

where the constants $\delta_{i}$ are to be found from the last equation. We compute

$$
Q_{i}=\left\langle p_{2}, A_{1}\left(q_{2}, s_{i}\right)+B\left(q_{2},\left(I_{n}-A\right)^{-1} J_{1} s_{i}\right)\right\rangle
$$

Copyright $@$ by SIAM. Unauthorized reproduction of this article is prohibited. 
for $i=1,2$. To obtain the derivative of the modulus and not the argument of the complex multiplier, we proceed as in [26, Appendix] but adapt to the case of maps. Then we find the following real solutions:

$$
\delta_{1}=\frac{\Re\left(e^{-i \theta_{0}} Q_{1}\right)}{\Re\left(e^{-i \theta_{0}} Q_{2}\right)}, \quad \delta_{2}=-\frac{1}{\Re\left(e^{-i \theta_{0}} Q_{2}\right)} .
$$

4. Algorithmic and numerical details. In this section we consider the computation of the derivatives and tensor-vector products, which are necessary not only for the continuation, but also for the computation of the critical normal form coefficients at codim 1 and 2 bifurcation points.

\subsection{Recursive formulas for derivatives of iterates of maps.}

4.1.1. Derivatives with respect to phase variables. The iteration of (1.1) gives rise to a sequence of points

$$
\left\{x^{1}, x^{2}, x^{3}, \ldots, x^{K+1}\right\},
$$

where $x^{J+1}=f^{(J)}\left(x^{1}, \alpha\right)$ for $J=1,2, \ldots, K$. Suppose that symbolic derivatives of $f$ up to order 5 can be computed at each point. We write

$$
A\left(x^{J}\right)_{i, j}=\frac{\partial f_{i}}{\partial x_{j}}\left(x^{J}\right), \quad B\left(x^{J}\right)_{i, j, k}=\frac{\partial^{2} f_{i}}{\partial x_{j} \partial x_{k}}\left(x^{J}\right), \quad C\left(x^{J}\right)_{i, j, k, l}=\frac{\partial^{3} f_{i}}{\partial x_{j} \partial x_{k} \partial x_{l}}\left(x^{J}\right),
$$

and similarly for $D\left(x^{J}\right)$ and $E\left(x^{J}\right)$.

We want to find recursive formulas for the derivatives of the composition (1.2), i.e., the coefficients of the multilinear functions in $(2.2)$ that we now denote by $A^{(J)}, B^{(J)}$, and $C^{(J)}$ to indicate the iterate explicitly:

$$
\left(A^{(J)}\right)_{i, j}=\frac{\partial\left(f^{(J)}\left(x^{1}\right)\right)_{i}}{\partial x_{j}}, \quad\left(B^{(J)}\right)_{i, j, k}=\frac{\partial^{2}\left(f^{(J)}\left(x^{1}\right)\right)_{i}}{\partial x_{j} \partial x_{k}}, \quad\left(C^{(J)}\right)_{i, j, k, l}=\frac{\partial^{3}\left(f^{(J)}\left(x^{1}\right)\right)_{i}}{\partial x_{j} \partial x_{k} \partial x_{l}},
$$

and $D^{(J)}$ and $E^{(J)}$ are analogously defined. What follows is a straightforward application of the chain rule.

For $J=1$ we have $A^{(1)}=A\left(x^{1}\right), B^{(1)}=B\left(x^{1}\right)$, and $C^{(1)}=C\left(x^{1}\right)$, and these are known. Now, as

$$
A_{i, j}^{(J)}=\sum_{k} \frac{\partial f_{i}}{\partial x_{k}}\left(f^{(J-1)}\left(x^{1}\right)\right) \frac{\partial\left(f^{(J-1)}\left(x^{1}\right)\right)_{k}}{\partial x_{j}}=\sum_{l} A\left(x^{J}\right)_{i, k} A_{k, j}^{(J-1)},
$$

we see that

$$
(F(x, \alpha))_{x}=A\left(x^{K}\right) A\left(x^{K-1}\right) \cdots A\left(x^{1}\right)-I_{n},
$$

where $F(x, \alpha)=f^{(K)}(x, \alpha)-x$.

For the second-order derivatives we first write $B^{(J)}$ once in coordinates

$$
\begin{aligned}
B_{i, j, k}^{(J)} & =\frac{\partial}{\partial x_{j}} \frac{\partial}{\partial x_{k}} f_{i}\left(f^{(J-1)}(x)\right) \\
& =\sum_{l, m} \frac{\partial^{2} f_{i}}{\partial x_{l} \partial x_{m}}\left(x^{J}\right) \frac{\partial\left(f^{(J-1)}\right)_{m}}{\partial x_{j}} \frac{\partial\left(f^{(J-1)}\right)_{l}}{\partial x_{k}}+\sum_{l} \frac{\partial f_{i}}{\partial x_{l}}\left(x^{J}\right) \frac{\partial^{2}\left(f^{(J-1)}\right)_{l}}{\partial x_{j} \partial x_{k}} .
\end{aligned}
$$

Copyright $\odot$ by SIAM. Unauthorized reproduction of this article is prohibited. 
For any two vectors $q_{1}$ and $q_{2}$, we can multiply the previous expression by $\left(q_{1}\right)_{j}\left(q_{2}\right)_{k}$ and sum over $(j, k)$ to obtain

$$
B^{(J)}\left(q_{1}, q_{2}\right)=B\left(x^{J}\right)\left(A^{(J-1)} q_{1}, A^{(J-1)} q_{2}\right)+A\left(x^{J}\right) B^{(J-1)}\left(q_{1}, q_{2}\right) .
$$

As $A\left(x^{J}\right)$ and $B\left(x^{J}\right)$ are known, (4.3) allows us to compute the multilinear form $B^{(J)}\left(q_{1}, q_{2}\right)$ recursively.

Let $q_{i}, i=1,2,3,4,5$, be given vectors. Multilinear forms with higher order derivatives can be computed with

$$
\begin{aligned}
C^{(J)}\left(q_{1}, q_{2}, q_{3}\right)= & C\left(x^{J}\right)\left(A^{(J-1)} q_{1}, A^{(J-1)} q_{2}, A^{(J-1)} q_{3}\right) \\
& +B\left(x^{J}\right)\left(B^{(J-1)}\left(q_{1}, q_{2}\right), A^{(J-1)} q_{3}\right)^{*} \\
& +A\left(x^{J}\right)\left(C^{(J-1)}\left(q_{1}, q_{2}, q_{3}\right)\right)
\end{aligned}
$$

where ${ }^{*}$ means that all combinatorially different terms have to be included, i.e.,

$$
\begin{aligned}
B\left(x^{J}\right)\left(B^{(J-1)}\left(q_{1}, q_{2}\right), A^{(J-1)} q_{3}\right)^{*}= & B\left(x^{J}\right)\left(B^{(J-1)}\left(q_{1}, q_{2}\right), A^{(J-1)} q_{3}\right) \\
& +B\left(x^{J}\right)\left(B^{(J-1)}\left(q_{1}, q_{3}\right), A^{(J-1)} q_{2}\right) \\
& +B\left(x^{J}\right)\left(B^{(J-1)}\left(q_{2}, q_{3}\right), A^{(J-1)} q_{1}\right) .
\end{aligned}
$$

For $D^{(J)}$ we get

$$
\begin{aligned}
D^{(J)}\left(q_{1}, q_{2}, q_{3}, q_{4}\right)= & D\left(x^{J}\right)\left(A^{(J-1)} q_{1}, A^{(J-1)} q_{2}, A^{(J-1)} q_{3}, A^{(J-1)} q_{4}\right) \\
& +C\left(x^{J}\right)\left(B^{(J-1)}\left(q_{1}, q_{2}\right), A^{(J-1)} q_{3}, A^{(J-1)} q_{4}\right)^{*} \\
& +B\left(x^{J}\right)\left(B^{(J-1)}\left(q_{1}, q_{2}\right), B^{(J-1)}\left(q_{3}, q_{4}\right)\right)^{*} \\
& +B\left(x^{J}\right)\left(C^{(J-1)}\left(q_{1}, q_{2}, q_{3}\right), A^{(J-1)} q_{4}\right)^{*} \\
& +A\left(x^{J}\right) D^{(J-1)}\left(q_{1}, q_{2}, q_{3}, q_{4}\right) .
\end{aligned}
$$

Finally, for $E^{(J)}$ the following holds:

$$
\begin{aligned}
E^{(J)}\left(q_{1}, q_{2}, q_{3}, q_{4}, q_{5}\right)= & E\left(x^{J}\right)\left(A^{(J-1)} q_{1}, A^{(J-1)} q_{2}, A^{(J-1)} q_{3}, A^{(J-1)} q_{4}, A^{(J-1)} q_{5}\right) \\
& +D\left(x^{J}\right)\left(B^{(J-1)}\left(q_{1}, q_{2}\right), A^{(J-1)} q_{3}, A^{(J-1)} q_{4}, A^{(J-1)} q_{5}\right)^{*} \\
& +C\left(x^{J}\right)\left(B^{(J-1)}\left(q_{1}, q_{2}\right), B^{(J-1)}\left(q_{3}, q_{4}\right), A^{(J-1)} q_{5}\right)^{*} \\
& +C\left(x^{J}\right)\left(C^{(J-1)}\left(q_{1}, q_{2}, q_{3}\right), A^{(J-1)} q_{4}, A^{(J-1)} q_{5}\right)^{*} \\
& +B\left(x^{J}\right)\left(C^{(J-1)}\left(q_{1}, q_{2}, q_{3}\right), B^{(J-1)}\left(q_{4}, q_{5}\right)\right)^{*} \\
& +B\left(x^{J}\right)\left(D^{(J-1)}\left(q_{1}, q_{2}, q_{3}, q_{4}\right)\right)\left(A^{(J-1)} q_{5}\right)^{*} \\
& +A\left(x^{J}\right)\left(E^{(J-1)}\left(q_{1}, q_{2}, q_{3}, q_{4}, q_{5}\right)\right) .
\end{aligned}
$$

The multilinear forms $A^{(K)}\left(q_{1}\right), B^{(K)}\left(q_{1}, q_{2}\right), C^{(K)}\left(q_{1}, q_{2}, q_{3}\right), D^{(K)}\left(q_{1}, q_{2}, q_{3}, q_{4}\right)$, and $E^{(K)}\left(q_{1}, q_{2}, q_{3}, q_{4}, q_{5}\right)$ are then used in the computations of the normal form coefficients for codim 1 and codim 2 bifurcations of period- $K$ cycles and also in branch switching.

Copyright $@$ by SIAM. Unauthorized reproduction of this article is prohibited. 
4.1.2. Derivatives with respect to parameters. If enough symbolic derivatives of $f$ are available, then MATCONT computes the expressions involving $J_{1}$ and $A_{1}$ in (2.2) symbolically. The idea is as follows. Taking the derivative of (1.3) with respect to $\alpha_{k}$ gives

$$
\frac{\partial\left(f^{(J)}\left(x^{1}, \alpha\right)\right)}{\partial \alpha_{k}}=\frac{\partial f}{\partial \alpha_{k}}\left(x^{J}, \alpha\right)+\frac{\partial f}{\partial x}\left(x^{J}, \alpha\right) \frac{\partial\left(f^{(J-1)}\left(x^{1}, \alpha\right)\right)}{\partial \alpha_{k}},
$$

which is recursively computable. Also mixed derivatives, which are necessary for continuation and branch switching, can be found recursively:

$$
\frac{\partial^{2}\left(f^{(J)}\left(x^{1}, \alpha\right)\right)}{\partial \alpha_{k} \partial x}=\frac{\partial^{2} f}{\partial \alpha_{k} \partial x}\left(x^{J}, \alpha\right)+\frac{\partial^{2} f}{\partial x^{2}}\left(x^{J}, \alpha\right) \frac{\partial\left(f^{(J-1)}\left(x^{1}, \alpha\right)\right)}{\partial \alpha_{k}} .
$$

In fact, the recursion is not applied to (4.8) itself, but to its product with a fixed vector.

This is sufficient for all continuations of fixed points and their codim 1 bifurcations. It is also sufficient for all cases of branch switching from codim 2 points, except for the case of degenerate flip. For this case, we fall back to a finite difference approximation. Since it is used only in the prediction step for which high accuracy is not needed, this seems acceptable.

4.2. Recursive formulas for derivatives of the defining systems for continuation. For the continuation of fixed points and cycles we need the derivatives of (1.3), which can be computed from (4.2) and (4.7).

Now, we consider the derivatives of $s$ (as defined in (2.4)) with respect to $z$, a state variable or parameter. The flip and NS cases can be handled in a similar way. Let $M$ be the matrix in (2.5). By taking derivatives of (2.5) with respect to $z$, we obtain

$$
M\left[\begin{array}{l}
v_{z} \\
s_{z}
\end{array}\right]+\left[\begin{array}{cc}
A_{z}^{(K)} & 0 \\
0 & 0
\end{array}\right]\left[\begin{array}{l}
v \\
s
\end{array}\right]=0
$$

Using (2.7), we obtain

$$
s_{z}=-w^{T}\left(A^{(K)}\right)_{z} v .
$$

If $z$ represents one of the state variables, then $s_{x_{i}}=-\left\langle w, B^{(K)}\left(e_{i}, v\right)\right\rangle$, as computed in section 4.1. When $z$ is a parameter $\alpha_{k}$ we can write

$$
s_{\alpha_{k}}=\sum_{J=1}^{K} C_{J},
$$

where

$$
C_{J}=-w^{T} f_{x}\left(x^{K}\right) \cdots\left(f_{x}\left(x^{J}\right)\right)_{\alpha_{k}} f_{x}\left(x^{J-1}\right) \cdots f_{x}\left(x^{1}\right) v,
$$

where $J=1, \ldots, K$. In this expression

$$
\left(f_{x}\left(x^{J}\right)\right)_{\alpha_{k}}=\left[f_{x}\left(f^{(J)}\left(x^{1}, \alpha\right)\right)\right]_{\alpha_{k}}=f_{x \alpha}\left(x^{J}, \alpha\right)+B\left(x^{J}\right) T_{J},
$$

where $T_{J}$ is a vector that can be recursively defined by

$$
T_{J}=f_{\alpha_{k}}\left(x^{J-1}, \alpha\right)+A\left(x^{J-1}\right) T_{J-1}, \quad T_{1}=0 .
$$

Summarizing, for the computation of $s_{\alpha}$ we need to compute $f_{x}, f_{\alpha_{k}}, f_{x x}, f_{x \alpha_{k}}$ in all iteration points $x^{1}, \ldots, x^{K}$, and given these, compute $T_{J}$ and $C_{J}$ for $J=1, \ldots, K$. 
4.3. Numerical computation of the directional derivatives. If symbolic derivatives of the original map are not available, then finite differences have to be used. However, the full tensors are not needed, but rather the multilinear forms evaluated on vectors, which can be computed with directional derivatives and central finite differences. This is an option of last resort and is not reliable for very high iterates. For a general discussion of directional derivatives, we refer to [18]. Similar to the analysis in [14, Appendix D], it can be shown that to compute a derivative of order $k$ the choice $h_{k}=\left(\epsilon_{m}\right)^{1 /(k+2)}$ for the stepsize minimizes the truncation and roundoff error under modest assumptions. Here $\epsilon_{m}$ denotes machine precision. In CL_MATCONTM, the Increment $\left(=h_{1}\right)$ can be adjusted by the user. The increments of the higher order derivatives are then adapted according to $h_{k}=\left(h_{1}\right)^{3 /(k+2)}$.

5. Examples. Here we give two examples illustrating the developed techniques and their implementation in CL_MATCONTM.

5.1. Generalized Hénon map. Consider the map

$$
F:\left(\begin{array}{c}
x_{1} \\
x_{2}
\end{array}\right) \mapsto\left(\begin{array}{c}
x_{2} \\
\alpha-\beta x_{1}-x_{2}^{2}+r x_{1} x_{2}
\end{array}\right),
$$

where $r$ is in principle not too large. This map appears in numerous theoretical studies of degenerate homoclinic bifurcations. A bifurcation analysis of this map can be found in [23] for $r=0$ and in [11] for general $r$. This map exhibits all planar codim 2 bifurcations with branch switching as in section 3.2. Let us start with the 1:2 resonance and the fold-flip. For these cases we can apply the algorithms analytically, i.e., with $r$ as a parameter. We note that $q=(1,-1)^{T}$ in both cases is an eigenvector of the Jacobian matrix corresponding to eigenvalue -1 .

For the 1:2 resonance we have the critical values $x_{1}=x_{2}=\frac{\beta-1}{r}$ and $\left(\alpha_{0}, \beta_{0}\right)=$ $\left(\frac{4(3-r)}{(2-r)^{2}}, \frac{2+r}{2-r}\right)$. For the coefficients of (3.6) the critical center manifold reduction yields (see [11])

$$
C_{1}=-\frac{1+r}{2}, \quad D_{1}=\frac{1}{4}\left(6+5 r+r^{2}\right) .
$$

Applying the algorithm from section 3.2.3, we find the prediction for the NS bifurcation curve of the period-2 cycle:

$$
\begin{gathered}
(x, y)=\left(\frac{2}{2-r}, \frac{2}{2-r}\right)+\sqrt{\frac{2 \varepsilon}{1+r}} q \\
(\alpha, \beta)=\left(\frac{4(3-r)}{(2-r)^{2}}, \frac{2+r}{2-r}\right)+\left(\frac{2\left(4+3 r^{2}-r^{3}\right)}{(1+r)(2-r)^{2}}, \frac{2 r^{2}}{(1+r)(2-r)}\right) \varepsilon .
\end{gathered}
$$

The fold-flip bifurcation occurs for $\left(x_{1}, x_{2}\right)=(0,0)$ at $\left(\alpha_{0}, \beta_{0}\right)=(0,-1)$. The critical center manifold reduction yields

$$
a=\frac{1}{2}(1-r), \quad b=\frac{1}{2}(1+r), \quad c_{2}=-\frac{1}{4}(1-r), \quad c_{4}=\frac{1}{4}(1+r)^{2} .
$$

Then applying the algorithm from section 3.2.6, we find the prediction for the NS bifurcation curve of the period-2 cycle emanating here:

$$
(x, y)=(0,0)+\sqrt{\frac{2 \varepsilon}{1+r}} q, \quad(\alpha, \beta)=(0,-1)+\left(2, \frac{-2 r^{2}}{(1+r)(2-r)}\right) \varepsilon .
$$


Let us compare the predictions with the exact expressions for these curves. Consider the following set:

$$
\begin{gathered}
\alpha=\frac{(1+\beta)\left(\beta-1-r+r^{2}\right)}{r^{2}} \\
x_{1,2}=\frac{r(\beta+1) \pm \sqrt{(r-2)(\beta+1)(2+r-\beta(2-r))}}{2 r}
\end{gathered}
$$

when

$$
\frac{1}{4}(3-r)(\beta+1)^{2} \leq \alpha \leq \frac{5+4 r+r^{2}+2 \beta(3+r)+\beta^{2}\left(5+2 r-r^{2}\right)}{2\left(2+2 r+r^{2}\right)} .
$$

It consists of two different pieces, where an NS bifurcation of a cycle of period 2 occurs. If we take $0 \leq \varepsilon \ll 1$ and consider the linear approximations of (5.4) near $\beta=-1-\frac{r^{2}}{2-r} \tilde{\varepsilon}$ and $\beta=\left(\frac{2+r}{2-r}\right)+\frac{r^{2}}{2-r} \tilde{\varepsilon}$, we find for the 1:2 resonance

$$
\begin{aligned}
& (\alpha, \beta)=\left(\frac{4(3-r)}{(2-r)^{2}}, \frac{2+r}{2-r}\right)+\left(\frac{4+3 r^{2}-r^{3}}{(2-r)^{2}}, \frac{r^{2}}{2-r}\right) \tilde{\varepsilon}+\mathcal{O}\left(\tilde{\varepsilon}^{2}\right), \\
& (x, y)=\left(\frac{2}{2-r}, \frac{2}{2-r}\right)+\sqrt{\tilde{\varepsilon}} q+\mathcal{O}(\tilde{\varepsilon}),
\end{aligned}
$$

and for the fold-flip bifurcation

$$
\begin{aligned}
& (\alpha, \beta)=(0,-1)+\left(1+r, \frac{-r^{2}}{2-r}\right) \tilde{\varepsilon}+\mathcal{O}\left(\tilde{\varepsilon}^{2}\right), \\
& (x, y)=(0,0)-\sqrt{\tilde{\varepsilon}} q+\mathcal{O}(\tilde{\varepsilon}) .
\end{aligned}
$$

So up to a positive factor our results coincide up to first order in $\varepsilon$.

For the other three cases a numerical approach is more illuminating. We now fix $r=-0.1$ and show in Figure 5.1(a) a (partial) bifurcation diagram in the parameters $\alpha, \beta$ computed with CL_MATCONTM. We pay closer attention to three specific regions, where we can switch branches: a spring area near $(\alpha, \beta)=(3,-1)$ with fold and PD curves with degeneracies, and two domains close to strong resonances near $\beta=1$. In the parameter space our predictions are linear, and for small initial amplitude the initialization routines worked well.

In Figure 5.1(b), there is a PD curve of period-4 cycles along which there are two degenerate flips near $(\alpha, \beta)=(3,-1)$, where we found a fold curve and a PD curve of cycles of period 4 . Then we produced the approximations to the fold curves of the eighth iterates in the degenerate flip points and from these easily continued the fold curves of cycles of period 8. The bifurcation structure of Figure 5.1(b) is rather universal; see [6]. In Figure 5.1(c) we show a neighborhood of the 1:3 resonance. For $r=-0.1$ the 1:4 resonance involves all of the mentioned local branches (see Figure 5.1(d)). In all subfigures we show the continuation results and also the approximate curves.

5.2. Leslie-Gower competition model. The origin of this example is in [21, $22,10]$. It has been found in biological experiments that two species of flour beetles can coexist under strong competition for the same food. This was rather unexpected at the time, and several models were built to explain this phenomenon. One of the 

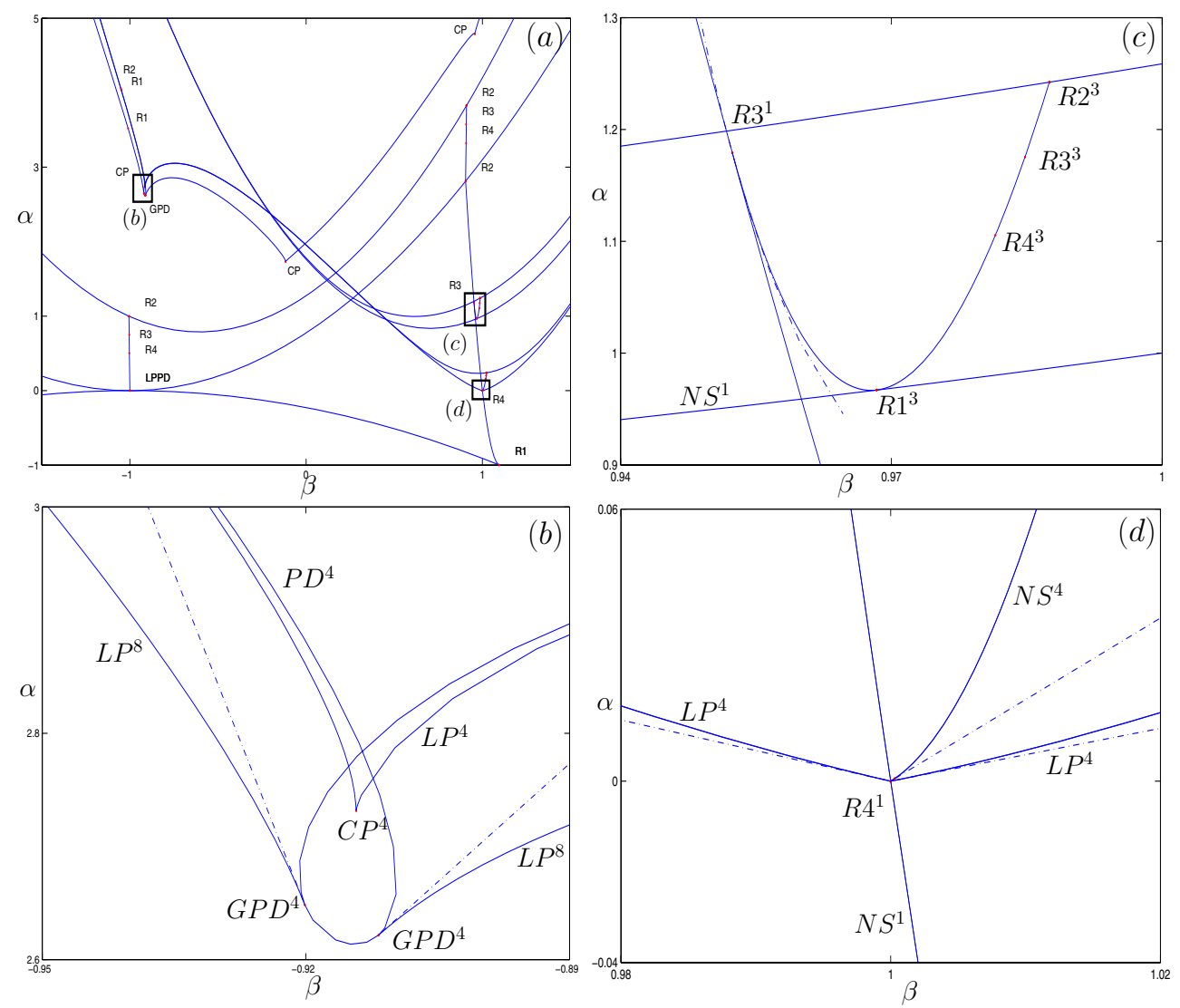

FIG. 5.1. Bifurcation diagram for the generalized Hénon map (a). The close-ups near a spring area of period 4 (b), 1:3 resonance of period 1 (c), 1:4 resonance of period 1 (d). The solid lines are computed with CL_MATCONTM; the dashed lines are the predictions from the switching algorithms.

ideas proposed in [10] and [25] was to use an age-structured population model. We consider the four-dimensional map model with 14 parameters in [25]:

$$
M_{L G}:\left(\begin{array}{l}
j \\
a \\
y \\
z
\end{array}\right) \mapsto\left(\begin{array}{l}
j^{\prime} \\
a^{\prime} \\
y^{\prime} \\
z^{\prime}
\end{array}\right)=\left(\begin{array}{c}
\frac{b_{1} a}{\left(1+c_{j j} j+c_{j a} a+c_{j y} y+c_{j z} z\right)} \\
\left(1-\mu_{j}\right) j+\left(1-\mu_{a}\right) a \\
\frac{b_{2} z}{\left(1+c_{y j} j+c_{y a} a+c_{y y} y+c_{y z} z\right)} \\
\left(1-\mu_{y}\right) y+\left(1-\mu_{z}\right) z
\end{array}\right) .
$$

This is the Leslie-Gower competition model for the interaction between the juveniles $(j)$ and adults $(a)$ of one species of the flour beetle Tribolium and the juveniles $(y)$ and adults $(z)$ of another species. Each species has its own juvenile recruitment rate $b_{1}>0, b_{2}>0$, juvenile death rates $\mu_{j}$ and $\mu_{y}$, and adult death rates $\mu_{a}$ and $\mu_{z}$. For biological reasons we have $0<\mu_{j}, \mu_{a}, \mu_{y}, \mu_{z}<1$.

The other coefficients $c_{j j}, c_{j a}, c_{j y}, c_{j z}$ and $c_{y j}, c_{y a}, c_{y y}, c_{y z}$ describe the competition. They are all strictly positive. By assumption, the competition does not affect the adults of either species. As in [25], we will study the influence of the coefficients 
TABLE 5.1

Parameter values for the Leslie-Gower model.

\begin{tabular}{|c|c|c|c|c|c|}
\hline$b_{1}=20$ & $c_{j j}=0.36$ & $b_{2}=18$ & $c_{j a}=0.55$ & $c_{j z}=0.23$ & $\mu_{j}=0.23$ \\
\hline$\mu_{a}=0.72$ & $c_{y a}=0.08$ & $c_{y y}=0.18$ & $c_{y z}=0.26$ & $\mu_{y}=0.29$ & $\mu_{z}=0.98$ \\
\hline
\end{tabular}

$c_{y j}$ and $c_{j y}$ on the behavior of $M_{L G}$ in a case where all other parameters are fixed. In other words, we study the role of the competition between juveniles alone.

The origin $(0,0,0,0)$ is a fixed point of $(5.6)$ but is of little interest. The model also has "horizontal" fixed points of the form $\left(j^{*}, a^{*}, 0,0\right)$, where $j^{*}, a^{*}>0$ (i.e., biologically meaningful) when $b_{1}\left(1-\mu_{j}\right)>\mu_{a}$. Similarly, there are unique "vertical" fixed points of the form $\left(0,0, y^{*}, z^{*}\right)$. These are biologically meaningful if $y^{*}, z^{*}>0$, i.e., when $b_{2}\left(1-\mu_{y}\right)>\mu_{z}$.

Furthermore there exists a unique coexistence fixed point $\left(j^{*}, a^{*}, y^{*}, z^{*}\right)$, provided

$$
H \equiv \delta \gamma-\epsilon \eta \neq 0,
$$

where

$$
\alpha=\frac{1-\mu_{j}}{\mu_{a}}, \quad \beta=\frac{1-\mu_{y}}{\mu_{z}},
$$

$\epsilon=c_{j j}+c_{j a} \alpha, \gamma=c_{j y}+c_{j z} \beta, \delta=c_{y j}+c_{y a} \alpha$, and $\eta=c_{y y}+c_{y z} \beta$. The equation $H=0$ defines a hyperbola in $\left(c_{y j}, c_{j y}\right)$ space. We will study the overall dynamics of the model for the parameter values specified in Table 5.1. The parameters $c_{j y}$ and $c_{y j}$ will vary. First we consider the horizontal and the vertical fixed points and their stability. For the given model parameters, the horizontal fixed point is stable if $c_{y j}>c_{y j 0}$, where $c_{y j 0}=0.474477674$. It is biologically plausible that the horizontal fixed point is stable only if the juveniles of the first species suppress the juveniles of the second species to a sufficient degree. The vertical fixed point is stable if $c_{j y}>c_{j y 0}$, where $c_{j y 0}=0.4571312026$.

Now we consider the coexistence fixed point $\left(j^{*}, a^{*}, y^{*}, z^{*}\right)$, starting the continuation from the point where $c_{y j}=c_{j y}=0$. This fixed point bifurcates into vertical and horizontal fixed points, respectively, when one of $c_{j y}$ and $c_{y j}$ is varied and the other variable is fixed at 0 . In the model this means that one species drives another to extinction. Continuation of the coexistence fixed point, where $c_{j y}$ is the free parameter, leads to $\mathrm{BP}$ and $\mathrm{PD}$ bifurcations at $c_{j y}=c_{y j 0}$ and $c_{j y}=0.170849$, respectively. The coexistence fixed point is stable before the $\mathrm{BP}$ and unstable afterwards. If we continue the coexistence fixed points with the free parameter $c_{y j}$, the points bifurcate into the horizontal fixed point at another BP. The coexistence fixed point is stable before this BP and unstable afterwards.

The solutions to the equation $H=0$, where $H$ is given by (5.7), are the parameter values for which the existence and uniqueness of the coexistence fixed point is not guaranteed. In the present context, where only $c_{y j}$ and $c_{j y}$ vary, this leads to the condition

$$
c_{y j} c_{j y}+a c_{y j}+b c_{j y}-c=0,
$$

where $a=0.1666326531, b=0.0855555552$, and $c=0.3350275226$, which indeed defines a hyperbola in $\left(c_{y j}, c_{j y}\right)$ space. It is not hard to prove that the point $\left(c_{y j 0}, c_{j y 0}\right)$ lies on the hyperbola. 


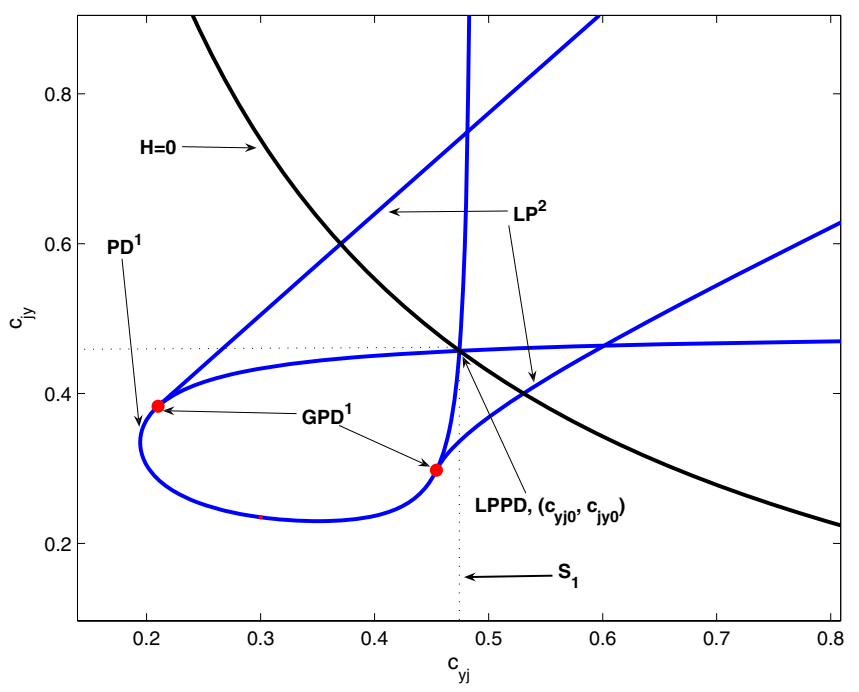

FIG. 5.2. The flip curve $P D^{1}$, the fold curve of the second iterate $L P^{2}$, hyperbola $H=0$, and the rectangle $S_{1}$ in the $\left(c_{y j}, c_{j y}\right)$-plane.

Now we return to the stability of the coexistence fixed points. The coexistence fixed point is unstable outside the rectangle

$$
S_{1}=\left\{\left(c_{y j}, c_{j y}\right): 0 \leq c_{y j} \leq c_{y j 0}, \quad 0 \leq c_{j y} \leq c_{j y 0}\right\} .
$$

Figure 5.2 shows the hyperbola $H=0$ and the rectangle $S_{1}$.

By numerical continuation we find that there is an interior region inside of $S_{1}$ in which the coexistence fixed points are unstable. This region is bounded by the $\mathrm{PD}$ curve, where the stability changes. The projection of the PD curve on the $\left(c_{y j}, c_{j y}\right)$-plane goes twice through the point $\left(c_{y j 0}, c_{j y 0}\right)$. Indeed, the PD curve has two fold-flip points, where $\left(c_{y j}, c_{j y}\right)=\left(c_{y j 0}, c_{j y 0}\right)$. Moreover, there are two degenerate PD points GPD on the PD curve: $\left(c_{y j}, c_{j y}\right)=(0.210138,0.383143)$ and $\left(c_{y j}, c_{j y}\right)=(0.454279,0.297779)$. The branches of fold curves of the second iterate can be computed by switching at the GPD points. These curves emanate tangentially to the PD curve.

The region where there are stable fixed points of the second iterate is bounded by the two fold curves of the second iterate and the lower left part of the PD curve. From the applications point of view, this is the most interesting region because it shows that indeed the two species can coexist even when the competition is strong. If both $c_{y j}$ and $c_{j y}$ are larger than 0.5 , then the horizontal fixed points, the vertical fixed points, and the fixed points of the second iterate are all stable. The PD curve and the fold curves of the second iterate are given in Figure 5.2.

6. Conclusions. We have described the implementation of continuation and normal form analysis of fixed points and cycles in MATCONT. Codim 1 bifurcation analysis is standard by now, while the analysis of codim 2 bifurcations, both quantitative and qualitative, on which this paper focuses, is new. Our implementation uses all aspects of the center-manifold reduction. First, the critical normal form coefficients are calculated automatically, which determines the type of the unfolding. Second, 
when nondegeneracy and transversality are checked, predictions about new codim 1 branches are made.

We use the iterates of maps to continue cycles. When dealing with strongly expanding/contracting systems and small basins of attraction, a BVP-like approach using extended systems for the fixed point, i.e., $f\left(x^{1}\right)-x^{2}=0, \ldots, f\left(x^{n}\right)-x^{1}=0$, might be more efficient.

Version 2.1 of CL_MATCONTM for maps uses automatic differentiation as an alternative to symbolic derivatives. One reason is that symbolic derivatives may not always be available. The second is that the computation of normal form coefficients for high iterates is faster when automatic differentiation is used.

Finally, we mention that we did not discuss global bifurcations. As a first step, it is well worth the effort to incorporate into our package some of the existing algorithms to compute (un)stable manifolds, e.g., [16]. If a transverse intersection is present, then using the defining systems as in [4], approximations of homoclinic orbits and their tangencies may be computed.

\section{REFERENCES}

[1] E. L. Allgower and K. Georg, Numerical Continuation Methods: An Introduction, SpringerVerlag, Berlin, 1990.

[2] V. I. ARnol'D, Geometrical Methods in the Theory of Ordinary Differential Equations, Springer-Verlag, New York, 1983.

[3] A. Back, J. Guckenheimer, M. Myers, F. Wicklin, and P. Worfolk, DsTool: Computer assisted exploration of dynamical systems, Notices Amer. Math. Soc., 39 (1992), pp. 303-309.

[4] W.-J. BEyn AND J.-M. KleinKauf, The numerical computation of homoclinic orbits for maps, SIAM J. Numer. Anal., 34 (1997), pp. 1207-1236.

[5] W.-J. Beyn, A. Champneys, E. Doedel, W. Govearts, Yu. A. Kuznetsov, and B. SANDSTEDE, Numerical continuation, and computation of normal forms, in Handbook of Dynamical Systems, Vol. 2, B. Fiedler, ed., Elsevier Science, Amsterdam, 2002, pp. 149-219.

[6] J.-P. Carcassés, C. Mira, M. Bosch, C. Simó, and J. C. Tatjer, "Crossroad area-spring area” transition. I. Parameter plane representation, Internat. J. Bifur. Chaos Appl. Sci. Engrg., 1 (1991), pp. 183-196.

[7] A. Dhooge, W. Govaerts, Yu. A. Kuznetsov, W. Mestrom, and A. M. Riet, CL_MATCONT: A continuation toolbox in MATLAB, in Proceedings of the 2003 ACM Symposium on Applied Computing, Melbourne, FL, 2003, Association for Computing Machinery, New York, pp. 161-166.

[8] A. Dhooge, W. Govaerts, and Yu. A. Kuznetsov, matCont: A MATLAB package for numerical bifurcation analysis of ODEs, ACM Trans. Math. Software, 29 (2003), pp. 141164.

[9] E. J. Doedel, A. R. Champneys, T. F. Fairgrieve, Yu. A. Kuznetsov, B. Sandstede, and X.-J. WANG, AUTO97-AUTO2000: Continuation and Bifurcation Software for Ordinary Differential Equations (with HomCont), User's Guide, Concordia University, Montreal, Canada (1997-2000), available online from http://indy.cs.concordia.ca.

[10] J. Edmunds, J. M. Cushing, R. F. Costantino, S. M. Henson, B. Dennis, And R. A. Desharnais, Park's Tribolium competition experiments: A non-equilibrium species coexistence hypothesis, J. Animal Ecology, 72 (2003), pp. 703-712.

[11] V. S. Gonchenko, Yu. A. Kuznetsov, and H. G. E. Meijer, Generalized Hénon map and bifurcations of homoclinic tangencies, SIAM J. Appl. Dyn. Sys., 4 (2005), pp. 407-436.

[12] W. Govaerts,Yu. A. Kuznetsov, and B. Sijnave, Bifurcations of maps in the software package CONTENT, in Computer Algebra in Scientific Computing-CASC'99 (Munich), V. G. Ganzha, V. G. E. W. Mayr, and E. V. Vorozhtsov, eds., Springer, Berlin, 1999, pp. 191-206.

[13] W. J. F. Govaerts, Numerical Methods for Bifurcations of Dynamical Equilibria, SIAM, Philadelphia, 2000.

[14] B. D. Hassard, N. D. Kazarinoff, And Y.-H. Wan, Theory and Applications of Hopf Bifurcation, Cambridge University Press, Cambridge, UK, 1981. 
[15] A. I. Khibnik, Yu. A. Kuznetsov, V. V. Levitin, and E. V. Nikolaev, Continuation techniques and interactive software for bifurcation analysis of ODEs and iterated maps, Phys. D, 62 (1993), pp. 360-371.

[16] B. Krauskopf and H. M. Osinga, Growing $1 D$ and quasi-2D unstable manifolds of maps, J. Comput. Phys., 146 (1998), pp. 404-419.

[17] Yu. A. Kuznetsov and V. V. Levitin, CONTENT: A Multiplatform Environment for Analyzing Dynamical Systems, CWI, Amsterdam, 1997; available online via ftp from ftp.cwi.nl/pub/CONTENT.

[18] Yu. A. Kuznetsov, Elements of Applied Bifurcation Theory, 3rd ed., Springer-Verlag, New York, 2004.

[19] Yu. A. Kuznetsov and H. G. E. Meijer, Numerical normal forms for codim 2 bifurcations of fixed points with at most two critical eigenvalues, SIAM J. Sci. Comput., 26 (2005), pp. 1932-1954.

[20] Yu. A. Kuznetsov and H. G. E. MeiJer, Remarks on interacting Neimark-Sacker bifurcations, J. Difference Equ. Appl., 12 (2006), pp. 1009-1035.

[21] P. H. Leslie And J. C. Gower, The properties of a stochastic model for two competing species, Biometrika, 45 (1958), pp. 316-330.

[22] P. H. Leslie, T. PARK, AND D. B. Mertz, The effect of varying the initial numbers on the outcome of competition between two Tribolium species, J. Animal Ecology, 37 (1968), pp. 9-23.

[23] C. Mira, Chaotic Dynamics, World Scientific, Singapore, 1987.

[24] H. Nusse And J. Yorke, Dynamics: Numerical Explorations, 2nd ed., Springer-Verlag, New York, 1998.

[25] Y. L. Song, The Juvenile/Adult Leslie-Gower Competition Model, preprint, 2004.

[26] D. Roose and V. HlavačEK, A direct method for the computation of Hopf bifurcation points, SIAM J. Appl. Math., 45 (1985), pp. 879-894.

Copyright (c) by SIAM. Unauthorized reproduction of this article is prohibited. 